\title{
Inventaire piscicole de la retenue hydroélectrique de Pareloup (Aveyron) lors de la vidange de juin 1993. Essai d'un nouveau système d'acquisition et de traitement des signaux d'un échosondeur
}

Fish inventory in the dammed reservoir of Pareloup (Aveyron, France) during draining in june 1993. Testing of a new system for acquisition and processing of echosound signals

\section{Richeux $\left(^{(*)}\right.$, J.F. Noguès $\left(^{(*)}\right.$, J.N. Toureng( ${ }^{(*)}$ et B. Aragon $\left(^{(*)}\right.$ \\ - Laboratoire d'hydrobiologie, URA 695 C.N.R.S., Université Paul Sabatier, 118 Route de Narbonne, F-31062 Toulouse Cedex, France. \\ * Laboratoire d'Acoustique, de Métrologie et d'Instrumentation, Université Paul Sabatier, 38 rue des 36 Ponts, F-31400 Toulouse Cedex, France.}

Résumé. - La vidange du lac de Pareloup, $5^{\ominus}$ retenue hydroélectrique de France (1260 ha, $169.10^{6} \mathrm{~m}^{3}$ ) a été l'occasion d'essayer de dresser un inventaire complet de sa faune piscicole. La méthode a consisté à récupérer les poissons dans une pêcherie spécialement aménagée à l'aval du barrage, estimer le nombre de poissons restés au niveau des ruisseaux par pêches électriques, ainsi que ceux réfugiés dans une conduite forcée, pêchés en cours de vidange et échoués dans le lac à sec.

Cette retenue, cyprinicole au moment de la vidange, abritait environ 89 tonnes de poissons de 15 espèces différentes largement dominées par les gardons (Rutilus rutilus (L.)) : 39,9 T. et les brèmes (Abramis brama (L.)) : 32,2 T. Trois carnassiers sont représentés en proportions égales : le brochet (Esox lucius L.) : 4,9 T., le sandre (Stizostedion lucioperca (L.)) : 4,6 T. et la perche (Perca fluviatilis L.) : 4,6 T.

Dans le cadre de la mise au point d'un systeme d'acquisition de signaux sonar léger, robuste et simple d'utilisation, la vidange a été l'occasion de mettre en pratique un système de localisation par satellites G.P.S. (Global Positioning System) utilisé en mode différentiel (précision du système différentiel réalisé : $<5 \mathrm{~m}$ à $95 \%$ ). Ceci a permis de suivre le mouvement des poissons par échosondage et G.P.S., de comparer les premières 
estimations obtenues par méthode acoustique avec le bilan de la vidange et d'apporter les modifications et réglages nécessaires au calibrage de l'instrumentation.

Mots-clés. - Inventaire piscicole, retenue artificielle, vidange, cyprinidé, carnassiers, pêche électrique, échosondage, G.P.S., comportement.

ABSTRACT. - The draining of Pareloup Lake, the fifth largest hydroelectric reservoir in France (1260 ha, $169.10^{6} \mathrm{~m}^{3}$ ), provided an opportunity to make a complete inventory of the fish fauna. The method consisted in recovering the fish in a fishery specially installed downstream, and estimating the number of fish remaining in small streams by means of electrofishing, as well as those taking shelter in a powerpipe gallery which were captured during the draining and those stranded in the dry lake.

This reservoir, mainly colonized by Cyprinidae at the time of the draining, contained some 89 tons of fish of 15 different species, largely dominated by roach (Rutilus rutilus [L.] - 39.9 tons) and bream (Abramis brama [L.] - 32.2 tons). Three carnivorous species were present in equal proportions: pike (Esox lucius L. -4.9 tons), pikeperch (Stizostedion lucioperca [L.] - 4.6 tons) and perch (Perca fluviatilis L.) - 4.6. tons).

In the framework of the fine-tuning of a lightweight acquisition system for sonar signals, both robust and easy to handle, the draining gave the occasion to implement the G.P.S. Global Positioning System using differential techniques (positioning accuracy of the differential system : < $5 \mathrm{~m}[95 \%]$ ). This enabled us to monitor fish movement by echosound and G.P.S., to compare the first estimations provided by acoustic methods with the inventory during the draining, and to make certain modifications and necessary adjustments in the calibration of the instrumentation.

Key-words. - Fish sampling, artificial reservoir, draining, Cyprinidae, carnivores, electrofishing, echosounding, G.P.S., behavior.

\section{INTRODUCTION}

Depuis sa mise en eau en 1951, la retenue hydroélectrique de Pareloup n'avait été vidangée qu'une seule fois en 1962. De nouvelles réfections au niveau du barrage s'imposaient et ont contraint EDF à évacuer la totalité de la masse d'eau (169 millions de $\mathrm{m}^{3}$ ).

En plus de 30 ans, un peuplement piscicole important s'est développé malgré des conditions climatiques parfois rigoureuses (amplitude thermique moyenne de l'eau: $20^{\circ} \mathrm{C}\left[2^{\circ}\right.$ $\left.22^{\circ} \mathrm{C}\right]$ ).

L'étude de la structure des populations en place s'avère d'un grand in- térêt en vue de la gestion de l'écosystème, mais également très problématique en raison des difficultés d'échantillonnage d'un lac d'une telle importance (1260 ha à la cote 805 NGF).

Les méthodes traditionnelles ne s'avèrent pas toujours suffisantes (Hartmann et al., 1987; Rose \& Leggett, 1988). Les études destinées à inventorier la faune pisciaire de Pareloup avaient toujours été effectuées avec des filets maillants de fond ou de surface (Dauba \& Tourenq, 1983; Béarez, 1988). Malgré l'emploi d'une large gamme de vide de maille (12 à $90 \mathrm{~mm}$ ), cette méthode de pêche utilisée seule ne peut garantir de résul- 
tats très fiables. Elle permet surtout de suivre une évolution des proportions des espèces. D'autres techniques comme la pêche au chalut, méthode lourde à mettre en œuvre, s'avèrent particulièrement délicates à Pareloup en raison de la morphologie du lac (fig. 1), ou comme les pêches à la senne en raison des nombreux rochers et branches qui jonchent le fond.

Les études montrent en fait que l'utilisation simultanée de plusieurs méthodes est indispensable pour estimer correctement un stock.

Ainsi, de nombreux chercheurs ont appliqué aux milieux lacustres les techniques acoustiques classiquement utilisées en océanographie, qu'ils couplent avec des pêches au filet (Bagenal et al., 1982; Burczynski \& Karp, 1985; Dahm, 1985; Hartmann et al., 1987; Jurvelius \& Heikkinen, 1987; Rose \& Leggett, 1988; Gerlotto, 1989; Blaxter \& Batty, 1990; Mathisen et al. cités par Thorne, 1990; Guillard, 1991; Walline et al., 1992; Degiogi et al., 1993).

Dans un but similaire, le Laboratoire d'Hydrobiologie (*) et le L.A.M.I. $\left(^{\star \star)}\right.$ se sont associés pour mettre au point un système d'acquisition de signaux sonar, simple d'utilisation, performant, robuste et transportable sur un zodiac.

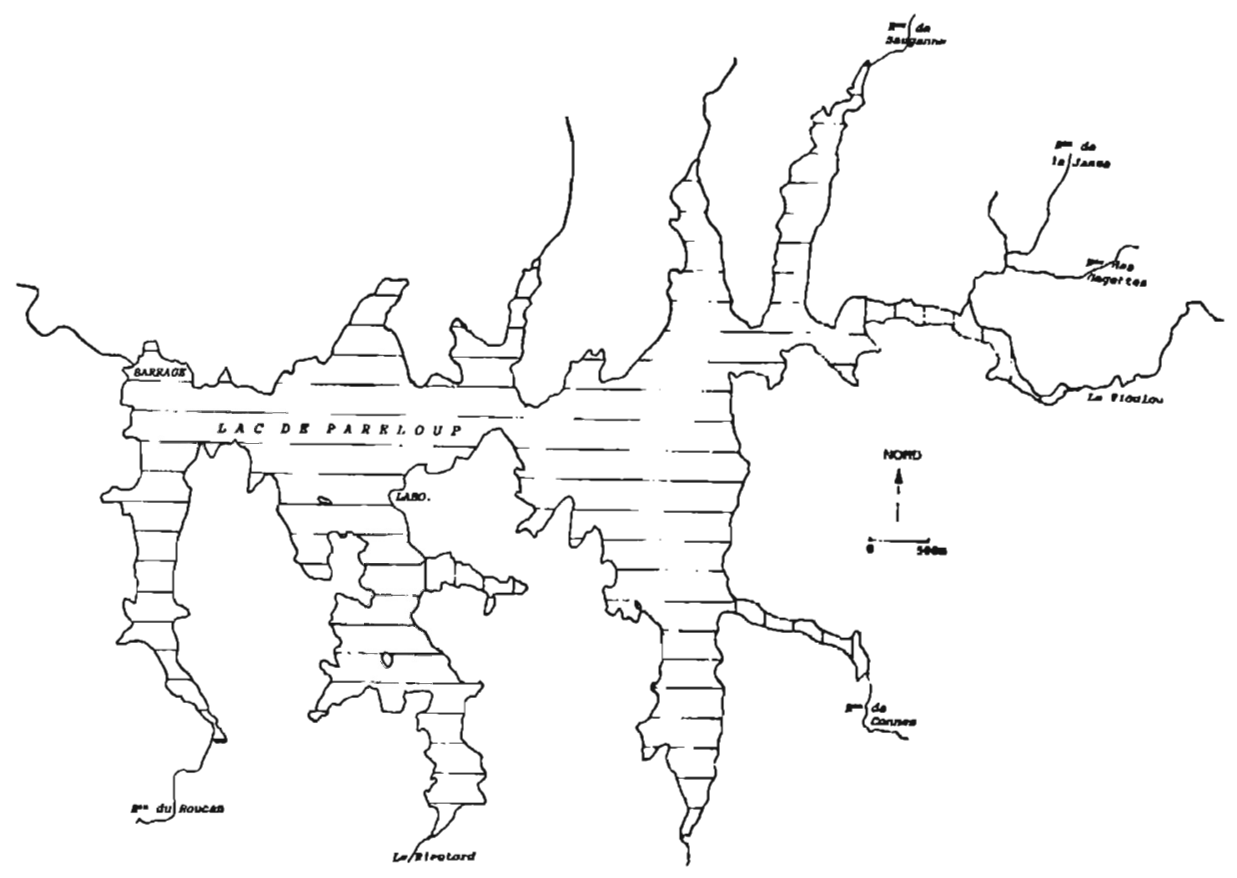

Fig. 1. - Plan théorique d'échantilonnage. Localisation des transects.

Fig. 1. - Theoretical sampling plan. Localization of transects. 
De gros progrès ont été réalisés ces dernières années, notamment grâce aux travaux de Salvetat (1991), et une instrumentation correspondant à nos objectifs est en passe de voir le jour. Ainsi, pour la première fois en janvier 1993, une couverture complète du lac a été réalisée en vue d'une première estimation du stock; l'expérience a été répétée à deux reprises (mars et juin) de manière à suivre le déplacement des poissons au cours de la baisse de niveau. L'opportunité de cette vidange devait nous permettre de vérifier la validité de nos estimations et de dresser un inventaire complet du stock grâce à une pêcherie située en aval du barrage.

\section{MATÉRIEL ET MÉTHODES}

\section{Campagnes hydroacoustiques}

\section{Principe de l'échosondage}

Une onde acoustique est émise verticalement dans le milieu aquatique. A chaque cible rencontrée, c'est à dire à chaque rupture d'impédance du milieu, une partie de cette onde est renvoyée vers l'émetteur (qui sert en général également de récepteur). C'est cet écho reçu qui est stocké et traité afin d'en extraire les informations relatives aux poissons ou au fond du lac.

La figure 2 illustre ce principe. Le transducteur transforme l'énergie électrique qu'il reçoit en énergie acoustique (émission). En réception, il effectue la conversion inverse et fournit un signal électrique relatif aux échos reçus.
L'échosondeur dont nous disposons est de type monofréquence $(192 \mathrm{KHz})$ monofaisceau $\left(16^{\circ}\right)$. II dispose d'un enregistreur papier permettant de visualiser le fond du lac et les poissons. II a été modifié afin d'en extraire directement les signaux électriques pour en effectuer l'acquisition et le traitement par un système plus performant.

\section{Système d'acquisition des don- nées}

L'enregistrement des données se fait grâce à un échointégrateur "Sibélius II" développé par le L.A.M.I. ("*).

Le système stocke des informations relatives au nombre de poissons détectés, à leur biomasse et répartition spatiale, et à la position de l'embarcation grâce au système de localisation par satellites (G.P.S.).

\section{Calibrage}

Le calibrage s'effectue par insonification d'une boule de cuivre de $32 \mathrm{~mm}$ de diamètre suspendue au dessous du transducteur le plus près possible de l'axe (Foote et al., 1987; Rose \& Leggett, 1988; Traynor et al, 1990; Guillard, 1991; Walline et al., 1992) à une profondeur de $2 \mathrm{~m}$.

\section{Plan d'échantillonnage}

Le lac a été échantillonné par transects parallèles équidistants (fig. 1) comme le suggèrent certains auteurs (Marchal \& Boely, 1977; Burczynski \& Karp, 1985; Jolly \& Hampton, 1990) à une vitesse constante de 4,9 ncuds. 


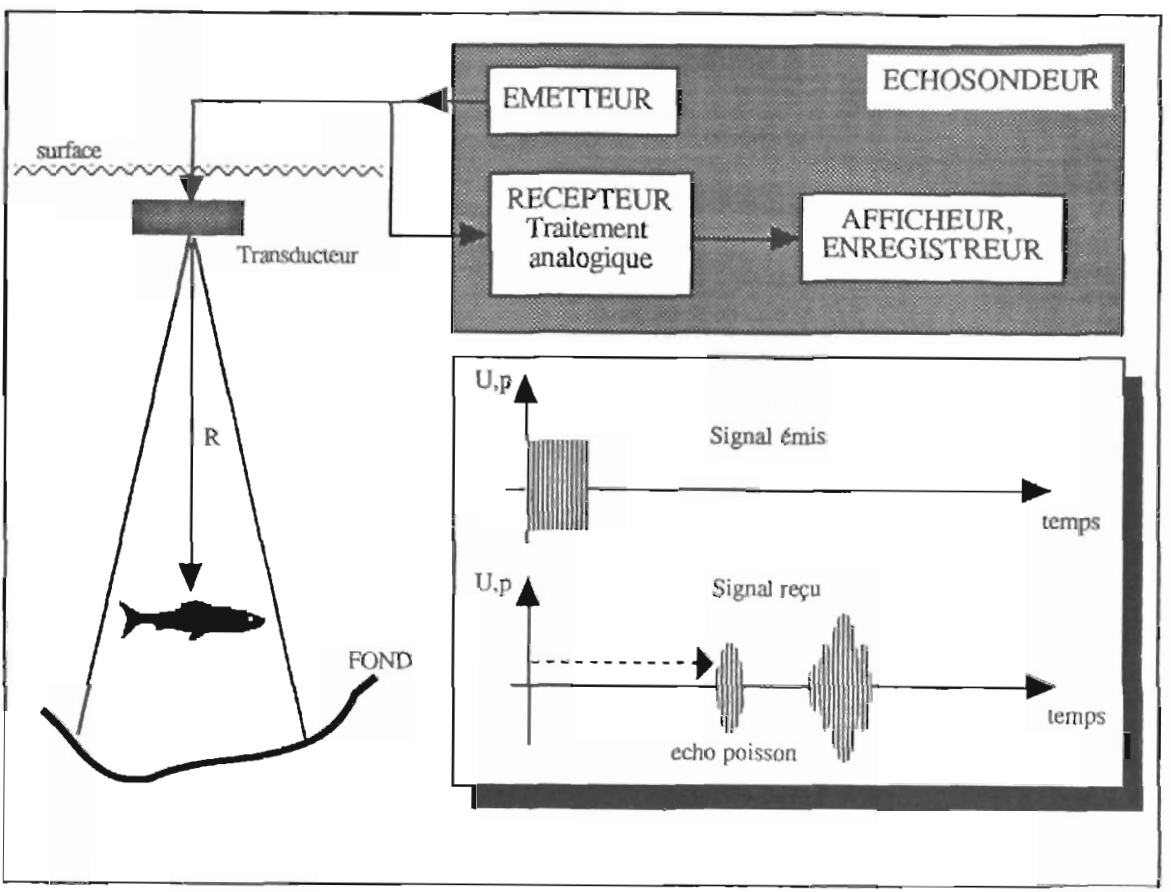

Fig. 2. - Principe de fonctionnement de l'échosondeur - représentation des signaux émis ot reçus. Fig. 2. - Principle of the echosounder - representation of emitted and received signals.

L'échantillonnage a eu lieu la nuit, lorsque les poissons sont le plus dispersés et donc mieux répartis en vue de leur dénombrement (Jurvelius et al., 1984; Laloë, 1985; Burczynski \& Karp, 1985; Jurvelius \& Heikkinen, 1987; Gerlotto, 1989; Guillard, 1991; Walline et al., 1992). Les migrations nycthémérales et les différences de répartition qui en résultent entraînent une sous-estimation lorsque la campagne est réalisée de jour (Bazigos, 1975; Jurvelius et al., 1987; Ponton \& Meng, 1990; Blaxter \& Batty, 1990).

Cependant, nous avons considéré qu'en raison du faible volume d'eau en juin (5,3 millions de $\left.\mathrm{m}^{3}\right)$, il n'y au- rait pas de différences jour/nuit significatives et la campagne a été menée de jour.

Dans tous les cas, nous avons opéré dans des conditions météorologiques favorables: sans vent, de manière à éviter toute turbulence source potentielle d'erreur (bullage en surface, faisceau en mouvement, etc.), et, lors des campagnes de nuit, par temps de pleine lune afin d'éviter l'utilisation d'une source lumineuse artificielle souvent à l'origine d'un changement de comportement du poisson à l'approche du bateau (Lévénez et al., 1990). 


\section{Analyse des résultats}

Les données enregistrées et stockées au niveau de l'échointégrateur sont ensuite transférées sur ordinateur. Les poissons apparaissent à l'écran comme des points à la position et la profondeur enregistrée. Les points sont colorés et codés pour indiquer le nombre de poissons détectés par tranche de $50 \mathrm{~cm}$ sur une distance de $10 \mathrm{~m}$ (photo 1). Cette représentation est extrêmement utile pour avoir un premier aperçu des données (Walline et al., 1992).

\section{Traitement des données}

Un traitement statistique "classique", par stratification, a été appliqué aux données de juin.

La variable utilisée est une densité moyenne de poissons détectés par $1000 \mathrm{~m}^{3}$. L'unité d'échantillonnage est le transect dans son ensemble. Les transects sont considérés comme répartis de façon aléatoire: en effet, si on a un dispositif d'échantillonnage systématique, il est choisi au hasard (distance inter-transect) et la position
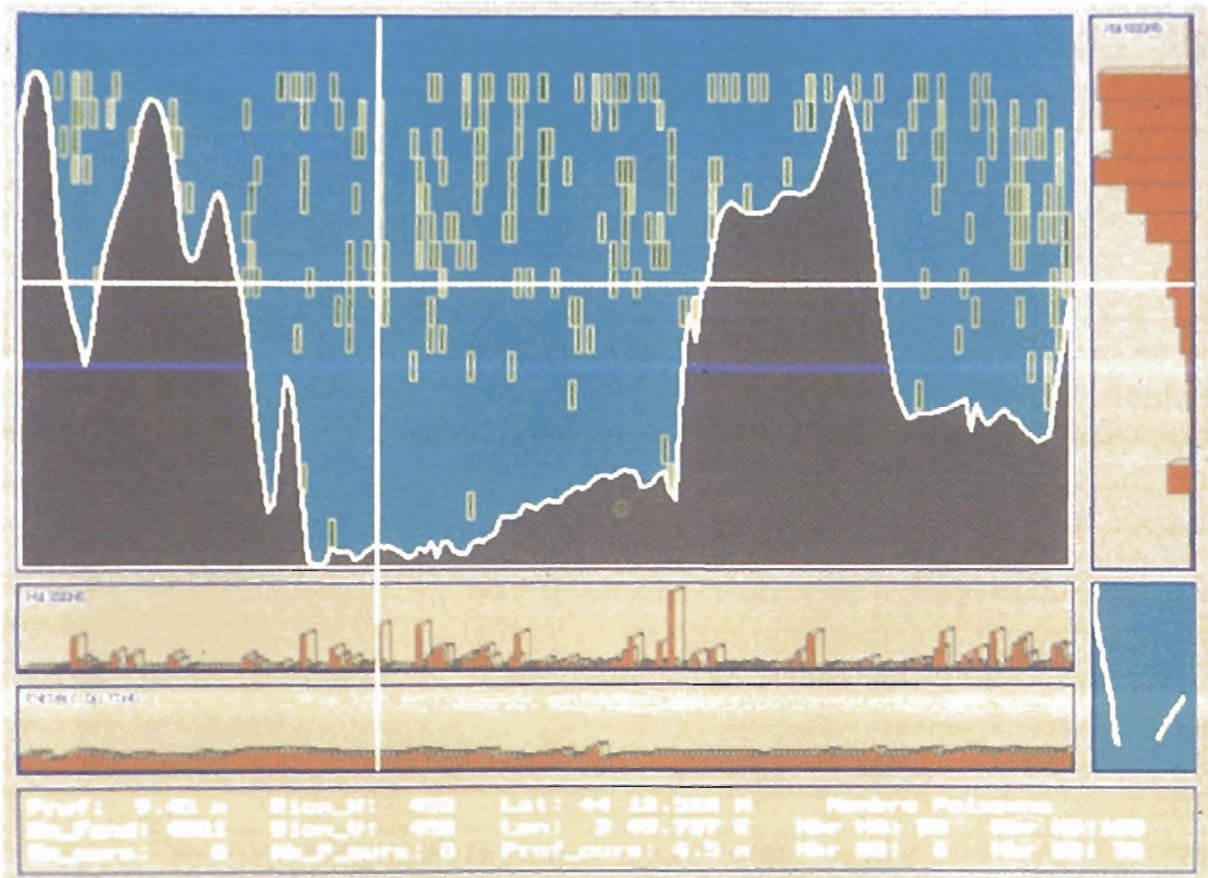

Loboratonie d'hudrobsologiw U.P.S. Toujausa

Photo 1. - Visualisation d'un transect sur écran d'ordinateur.

Photo 1. - Computer display of a transect. 
sur le lac ne nous permet pas d'avoir une précision telle que chaque transect soit à une distance rigoureusement identique au suivant.

Cette méthode est rapide et peu coûteuse en temps de calcul (Williamson, 1982; Traynor et al., 1990; Guillard, 1991), mais présente l'inconvénient d'être moins précise car on obtient des intervalles de confiance très larges dûs au fait que les variances des échantillons sont très fortes. Ceci explique que, traditionnellement, les approches par stratification sont employées sans la prise en compte de la variance de l'échantillonnage (Marchal \& Boely, 1977; Juvelius et al., 1984).

Toutefois, nous avons retenu cette méthode dans laquelle les poissons sont comptés par strate de $2 \mathrm{~m}$, le nombre total correspondant à la somme des strates. Considérant que les densités moyennes par strate sont indépendantes et que la distribution suit une loi normale Gaussienne, le calcul de la population totale a été effectué à partir des paramètres suivants :

$$
\text { 1) } \bar{Y}=\sum_{h=1}^{K} W h * \bar{Y} h
$$

où $\bar{Y}$ est un estimateur de la moyenne de la population statistique.

Wh est le poids de la strate $h$ :

$$
W h=\frac{N h}{N}
$$

$\mathrm{Nh}=$ Volume de la strate de profondeur $h$

$\mathbf{N}=$ Volume total du lac
$\bar{Y} h$ est la densité moyenne de la strate h:

$$
\overline{Y h}=\frac{\sum_{i=1}^{n h} Y h i}{n h}
$$

$\sum_{i=1}^{n h} Y h i=$ densité de poissons

$\mathrm{nh}=$ effectifs de l'échantillon $\mathrm{h}$

2) $\hat{Y}=N \bar{Y}$

où $\hat{Y}$ est un estimateur de la population totale du lac

3) $v(\hat{Y})=N^{2} v(\bar{Y})$

où $v(\hat{Y})$ est la variance de la population du lac et $v(Y)$ est la variance de la population statistique et se calcule comme suit :

$$
v(\bar{Y})=\sum_{h=1}^{K} \frac{W^{2} h * S^{2} y h}{n h}
$$

$S^{2} y h$ est la variance de l'échantillon de la strate $h$ et se calcule ainsi :

$$
S^{2} y h=\frac{\sum_{i=1}^{n h}(Y h i-\bar{Y} h)^{2}}{n h-1}
$$

4) L'intervalle de confiance à $95 \%$ de la population du lac a finalement a finalement été déterminé ainsi :

$$
\begin{aligned}
\operatorname{Pr}\left\{\hat{\mathrm{Y}}-\mathrm{t}_{.05 / 2}\right. & \operatorname{Vv}(\hat{\mathrm{Y}})<\mathrm{Y}<\hat{\mathrm{Y}} \\
& \left.+\mathrm{t}_{.05 / 2} \operatorname{VV}(\hat{\mathrm{Y}})\right\}=1-\alpha
\end{aligned}
$$

L'intervalle de confiance à $95 \%$ fournit les limites à l'intérieur desquelles le paramètre de la population Y a $95 \%$ de chance de se trouver. 


\section{Déroulement de la vidange}

Le niveau a progressivement été baissé en 3 étapes (fig. 3) : une première baisse nette a eu lieu entre janvier et mars, une seconde entre mars et juin, par turbinage de l'usine d'Alrance (retenue de Villefranche de Panat, fig. 4) et enfin, évacuation du reste de l'eau par ouverture des vannes de fond le 14 juin à 8 h20.

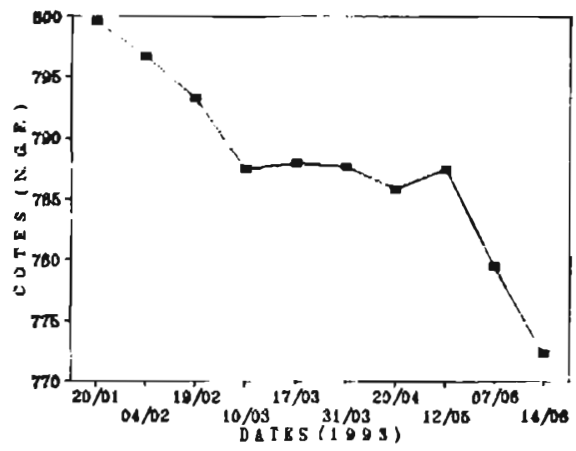

Fig. 3. - Evolution de la cote du lac les 6 mois précédant la vidange.

Fig. 3. - Variation in water level in Pareloup for 6 months preceding the draining.
C'est par cette voie que la majorité des poissons s'est trouvée entraînée vers une pêcherie construite pour la circonstance et située à environ $300 \mathrm{~m}$ en aval du barrage.

\section{Principe de la pêcherie}

Le principe de la pêcherie était de guider tout le débit de la rivière sur un plancher surélevé par rapport au lit de celle-ci. Ce plancher était constitué de barreaux de section rectangulaire espacés de $1,5 \mathrm{~cm}$, calculé pour pouvoir supporter une vingtaine de personnes.

Les fines, sables et graviers pouvaient donc passer tandis que les poissons, bien visibles, pouvaient être récupérés (photo 2).

Les deux tiers de la surface du plancher étaient inclinés en pente douce vers l'amont $(7 \%)$ de maniere à favoriser l'échouage des poissons emportés sur le front de la lame d'eau.

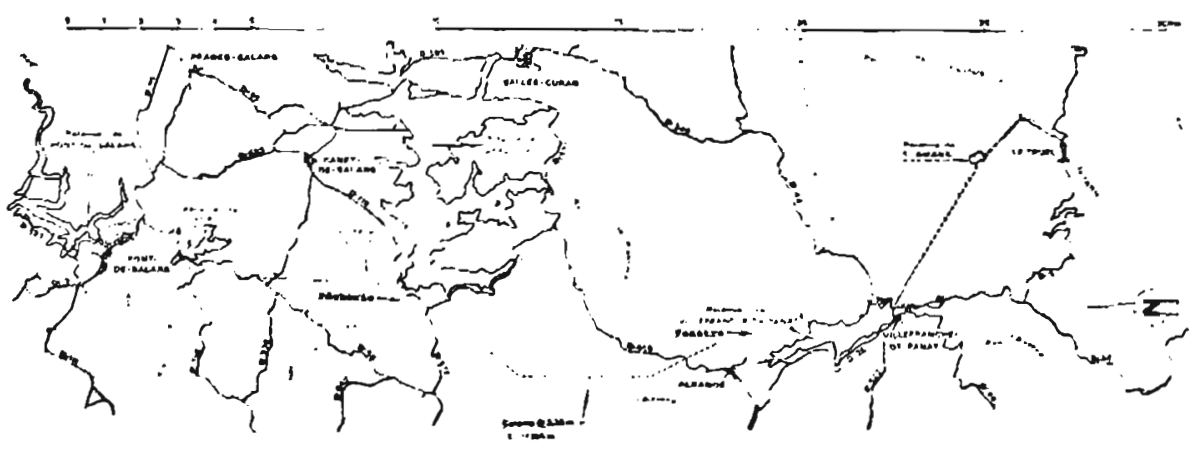

Fig. 4. - Aménagement hydrographique.

Fig. 4. - Hydrographic installation. 


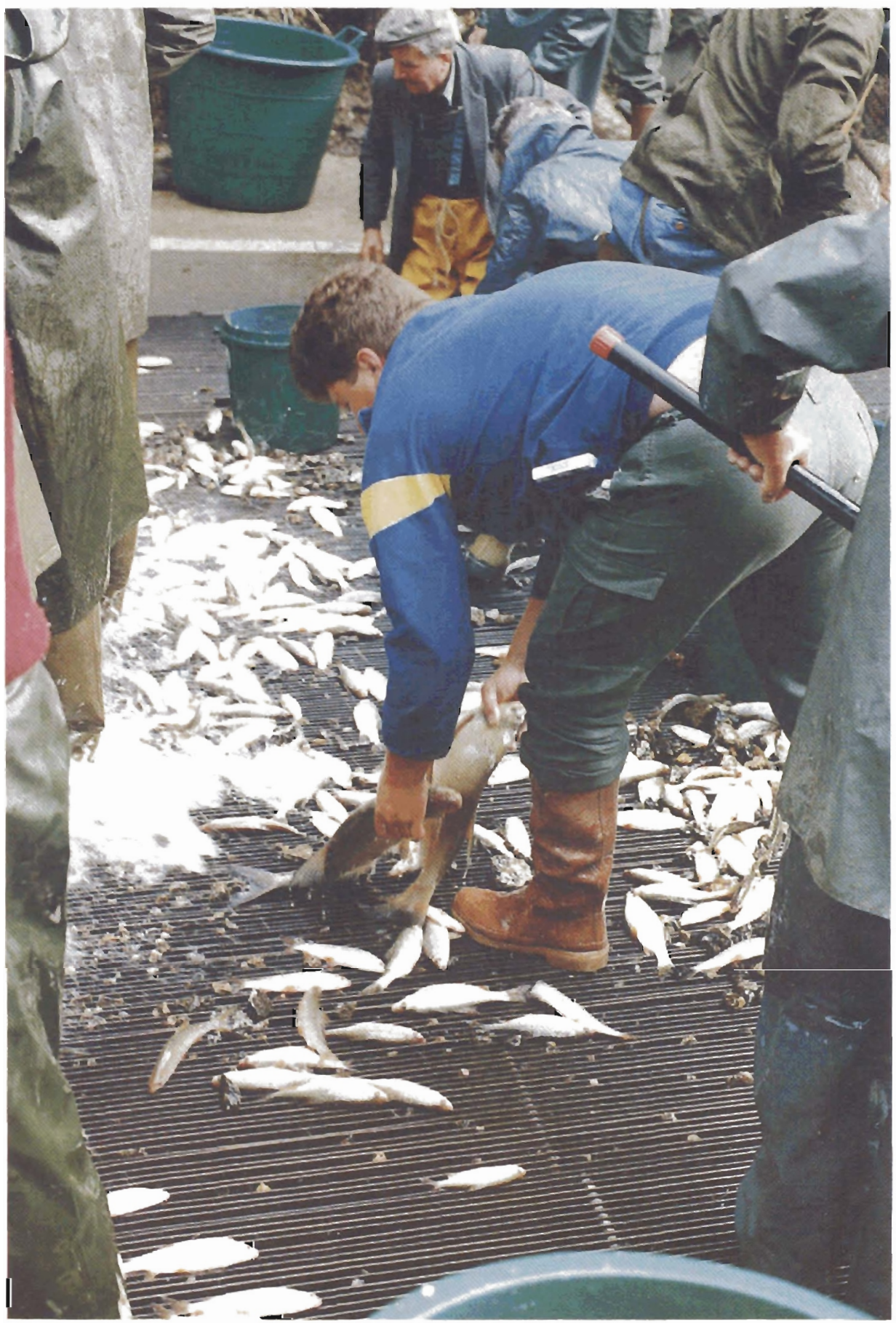

Photo 2. - Récupération des poissons au niveau de la pêcherie.

Photo 2. - Recovery of fish at the downstream fishery. 


\section{Récupération des poissons au niveau de la pêcherie}

A ce niveau, les poissons sont récupérés (photo 2), remontés vers un centre de tri (photo 3), mesurés par espèce (photo 4) et comptés. Puis des étalonnages de poubelles visant à estimer le poids d'une poubelle pleine, le nombre de poissons contenus et les proportions des différentes espèces ont été effectués. Ces opérations ont été réalisées en continu les 30 premières heures de la vidange. On a pu ainsi définir des poubelles à "dominante gardons" et des poubelles à "dominante brèmes". Les proportions ainsi établies ont été considérées constantes pour les 4 jours de la vidange, l'arrivée massive de poissons à partir de la $30^{\circ}$ heure n'ayant pas permis des étalonnages de contrôle. Puis, les poubelles ont été dénombrées dans leur ensemble, seuls les brochets, sandres, carpes, tanches, truites et chevesnes ont fait l'objet d'un dénombrement individuel pendant toute la vidange.

Les brèmes ont été sacrifiées et stockées dans des bennes, ainsi que l'ensemble des poissons morts, en vue de la fabrication de farine de poisson (photo 5).

Les poissons vivants ont été récupérés, comptés et stockés dans des bacs de stabulation (photo 6) avant d'être transportés dans d'autres lacs et rivières.
Récupération des poissons au niveau de la conduite forcée

De nombreux poissons se sont réfugiés dans la galerie reliant Pareloup à l'usine d'Alrance $(11,3 \mathrm{~km})$ lors de la vidange (fig. 4). La récupération des poissons a nécessité l'emploi d'un tracto-pelle adapté introduit dans le canal qui a permis de "pousser" les poissons vers un point de récupération (fig. 4, photo 7).

Sur le même principe qu'au niveau de la pêcherie, les poissons ont été remontés dans des poubelles et leur nombre et proportions établis. Puis les poubelles ont été comptées et les poissons vivants récupérés.

\section{Dénombrement des poissons au niveau des ruisseaux}

Le lac vidé, les ruisseaux (Vioulou, Connes, Roucan...) ont retrouvé leurs anciens lits (photo 8). Le nombre de poissons restés à ce niveau a été estimé grâce à des pêches électriques réalisées en différents points (fig. 5 , photo 9).

Les poissons ont été comptés, mesurés et pesés par secteur de 80 à $500 \mathrm{~m}$, puis le nombre total estimé par extrapolation à la longueur totale des cours d'eau jalonnant le lac à sec.

\section{Estimation du nombre de poissons pêchés lors de la vidange}

Des autorisations spéciales ont permis la pêche pendant la vidange. Une 


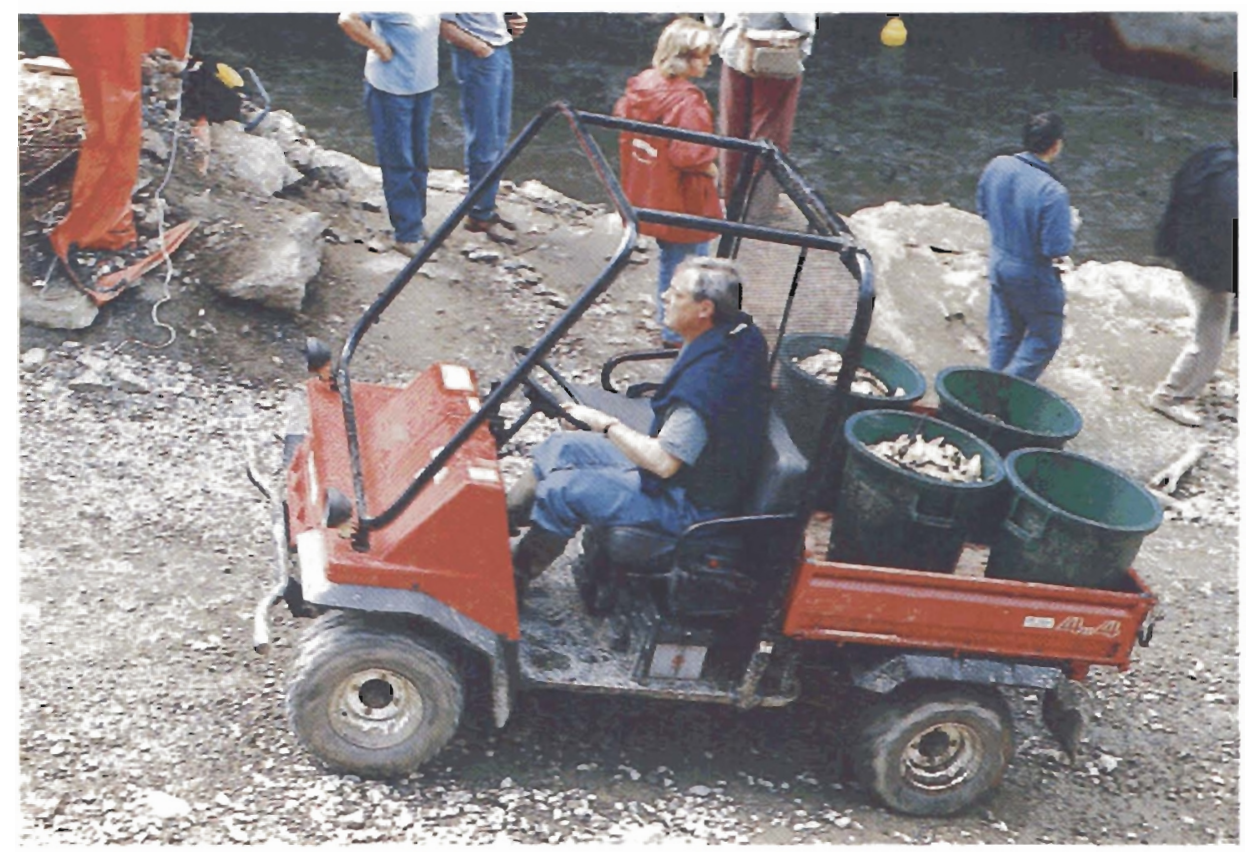

Photo 3. - Centre de tri.

Photo 3. - Sorting center.

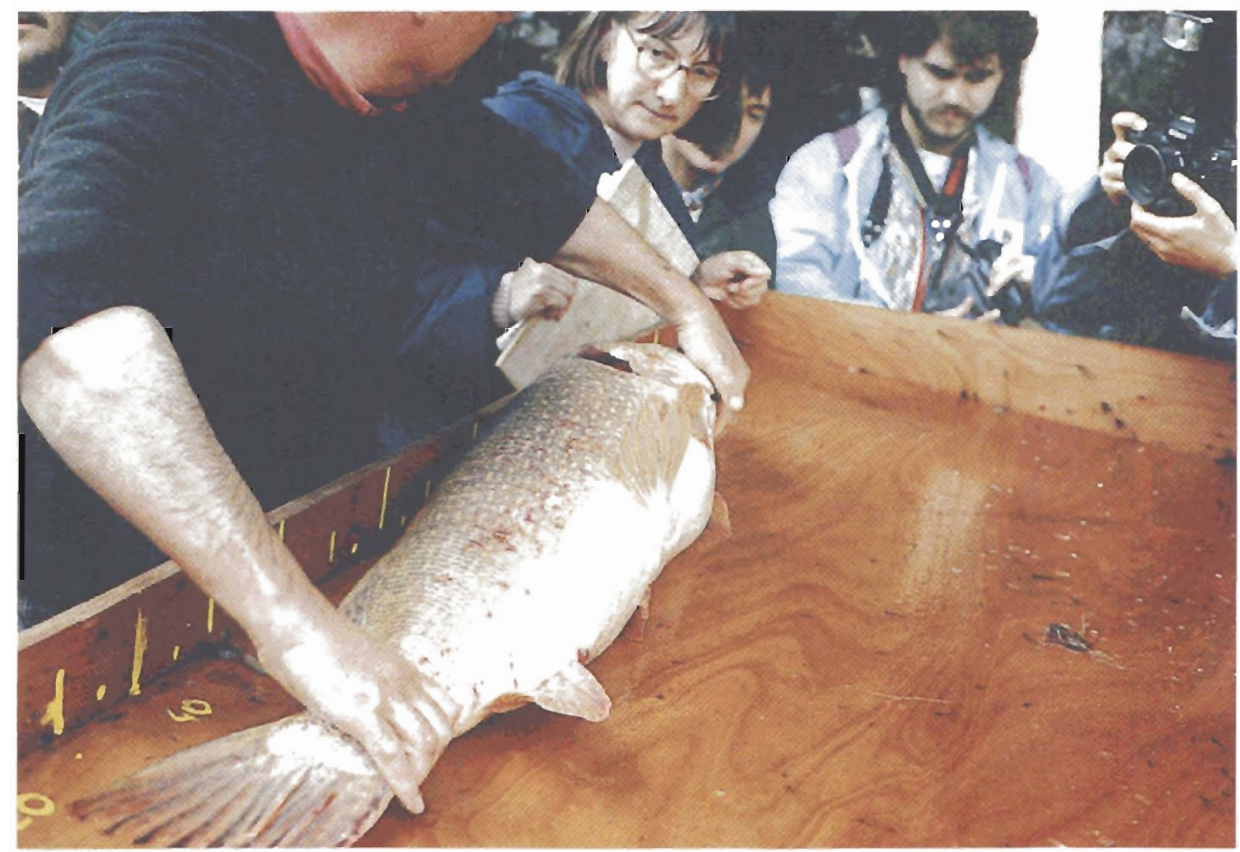

Photo 4. - Mensuration des poissons par espèce.

Photo 4. - Measurement of fish according to species. 


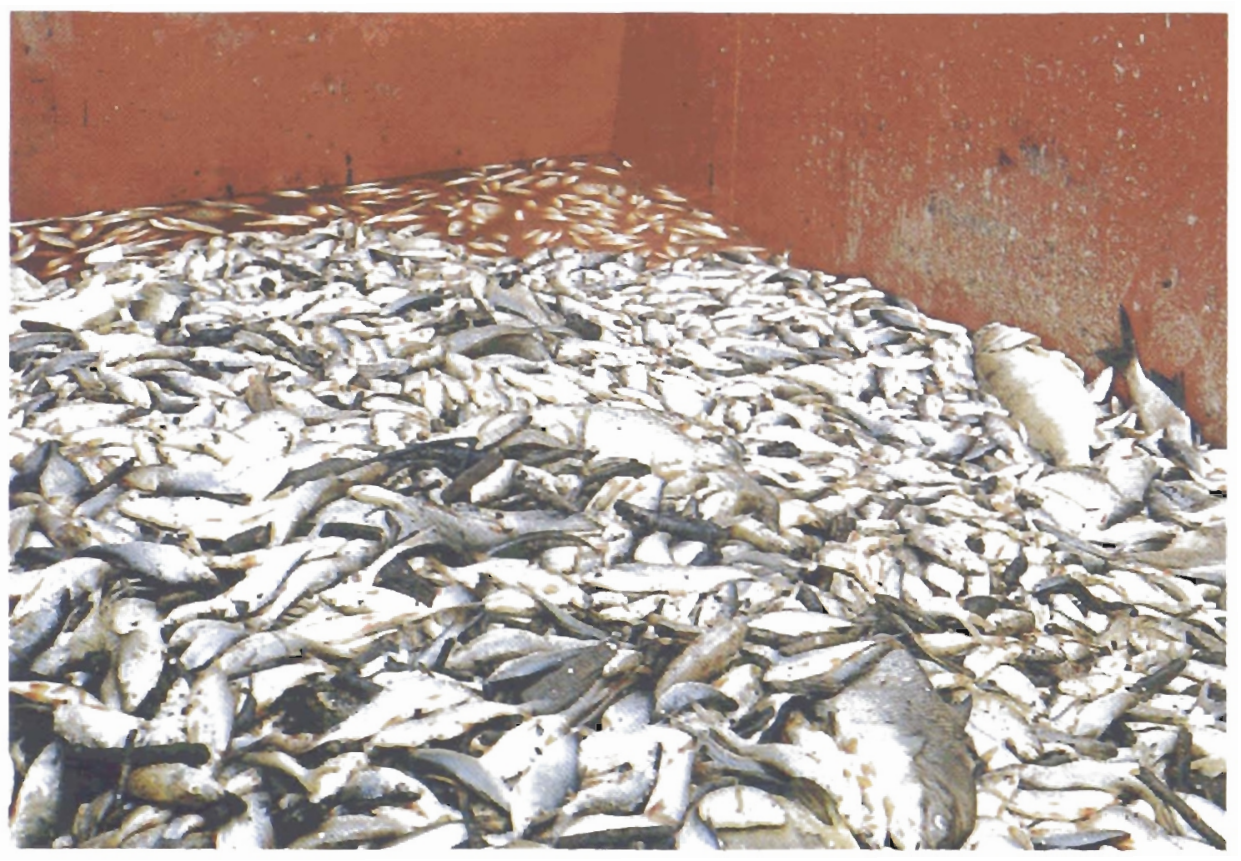

Photo 5. - Benne recueillant les poissons morts.

Photo 5. - Container for collection of dead fish.

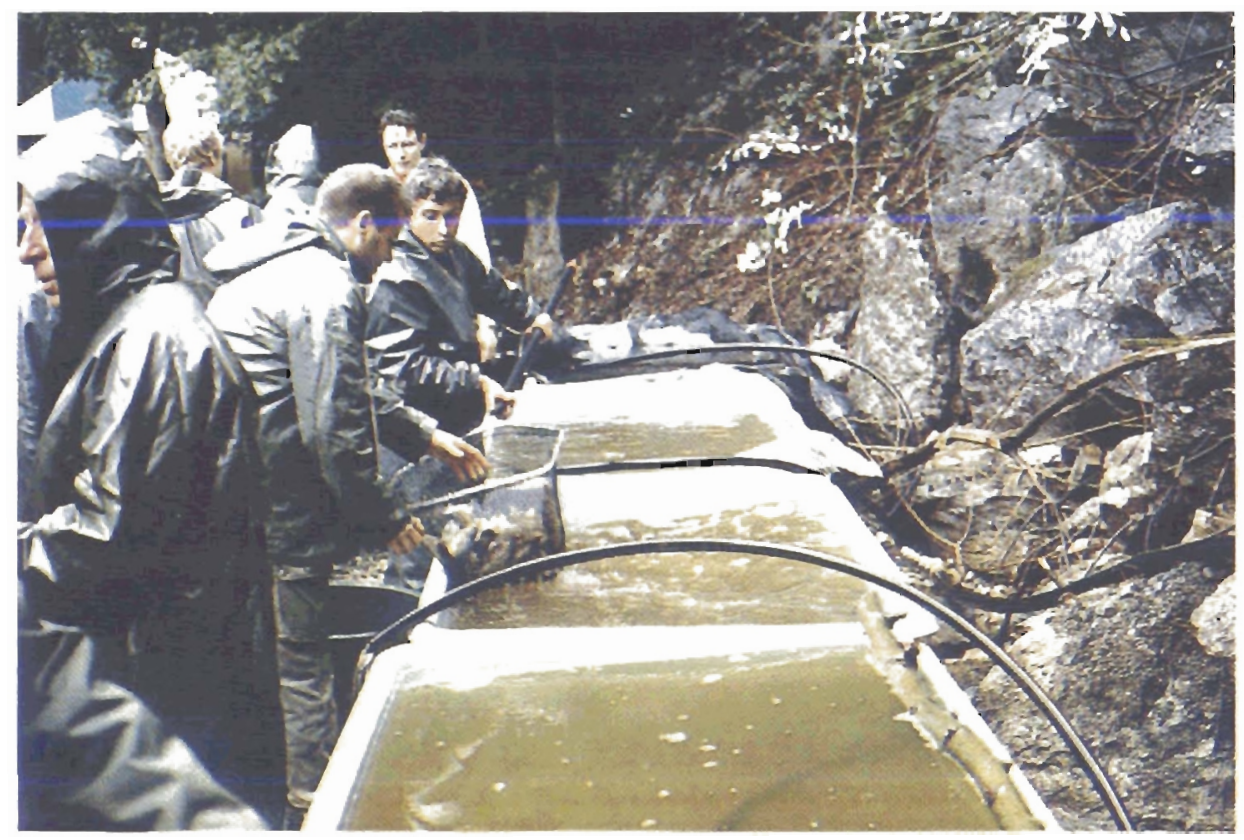

Photo 6. - Bac de stabulation.

Photo 6. - Recuperation basin. 


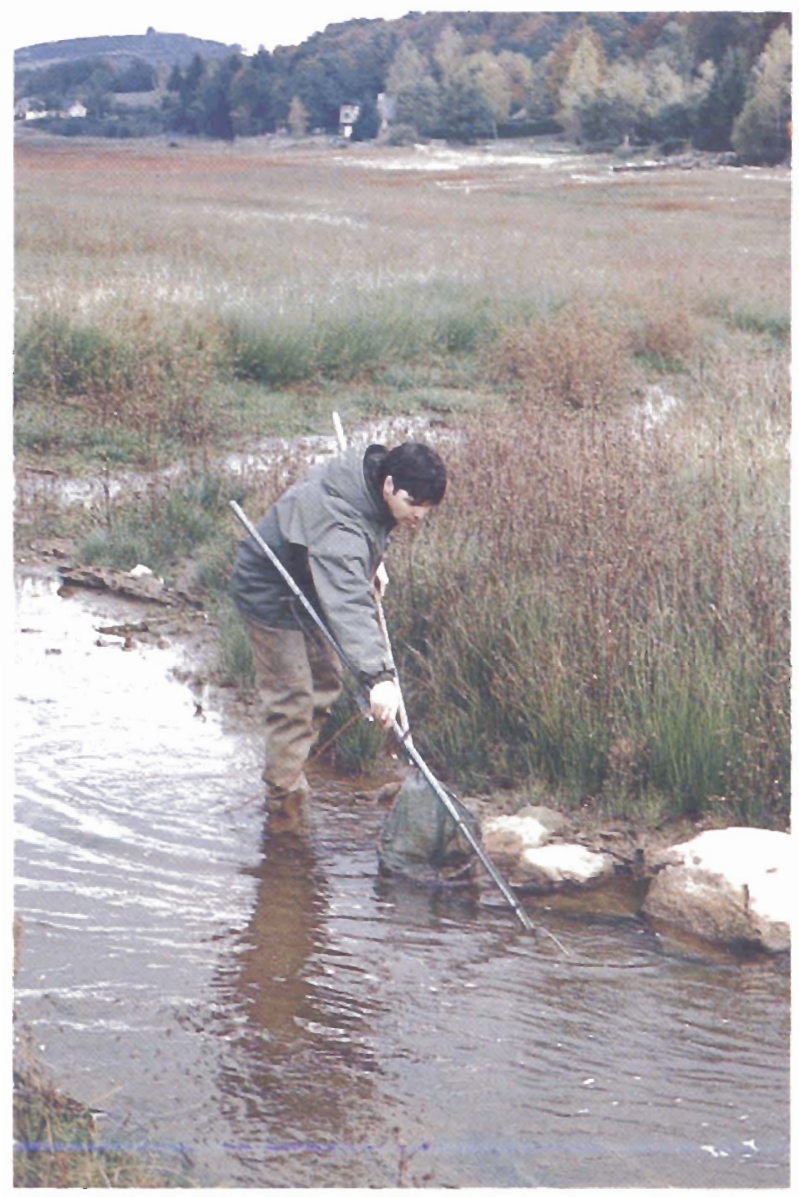

Photo 9. - Pêche électrique.

Photo 9. - Electrofishing.

enquête menée par Monsieur J.M. Nayral auprès des autochtones a été la seule façon d'estimer la quantité de poissons pêchés en amont du barrage.
Evaluation du nombre de poissons échoués

Cette estimation est problématique en raison des difficultés d'accès au niveau du lac liées à l'instabilité du 


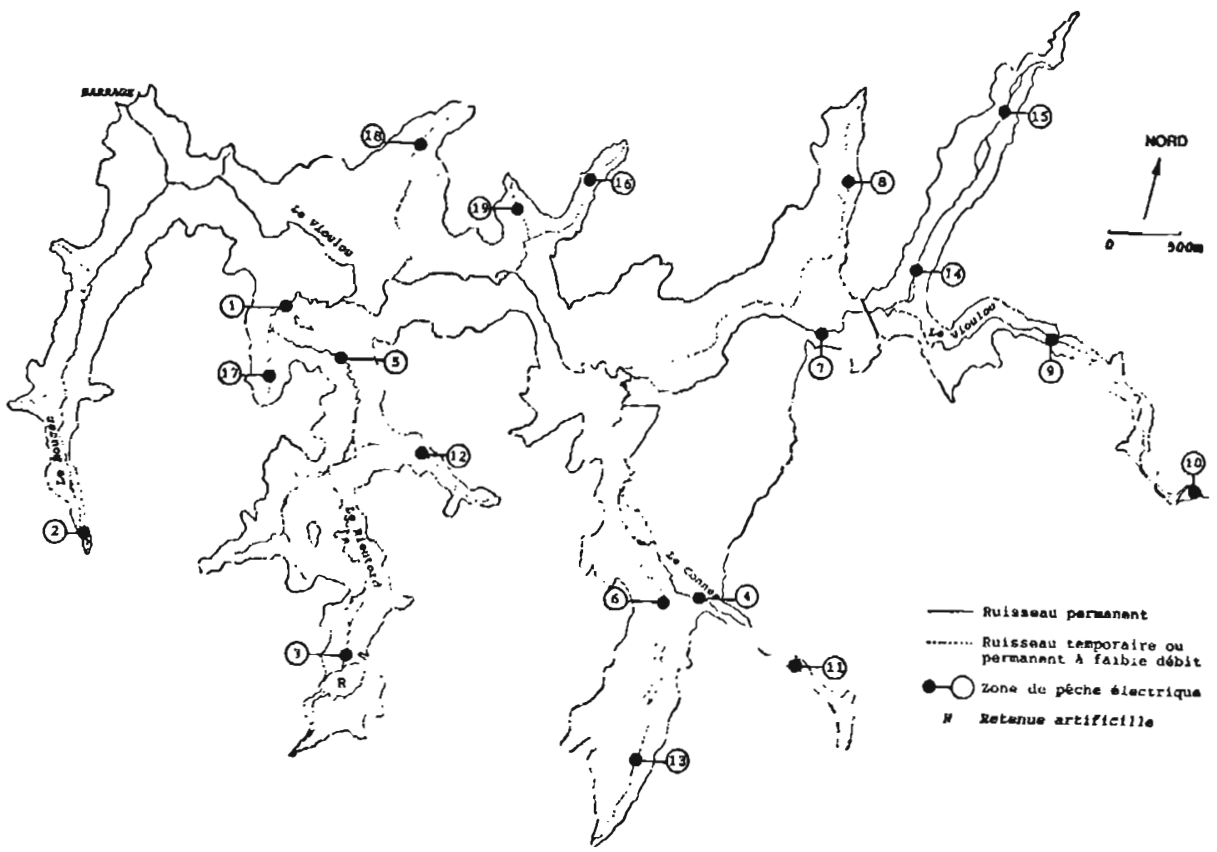

Fig. 5. - Carte des ruisseaux parcourant le lac après la vidange. (Carte réalisée à partir de photos aériennes: mission SPHAIR NB 1/9000 du 08/07/93).

Fig. 5. - Map of streams through the lake after the draining. (Map drawn up from aerial photos. - SPHAIR NB $1 / 9000$ mission of July 8, 1993.)

substrat encore gorgé d'eau. Ce n'est qu'à partir d'observations (estimation du nombre de poissons échoués par $\mathrm{m}^{2}$ ) que nous avons tenté d'estimer ce nombre.

\section{RÉSULTATS}

\section{Campagnes d'échosondage}

Les différentes campagnes réalisées entre janvier et juin 1993 ont permis de suivre la répartition du poisson au fur et à mesure de la baisse de niveau et de donner une première estimation du stock (en nombre).

\section{Répartition horizontale}

Le résultat d'une campagne d'échosondage se présente comme un ensemble de densités, exprimées en nombre de poissons par $1000 \mathrm{~m}^{3}$ (ou pour la biomasse en unités d'échointégration), localisées dans l'espace.

Des cartes de visualisation des résultats ont été dressées (fig. 6, 7, 8) et représentent des valeurs de densités pour des séquences d'environ $80 \mathrm{~m}$; les cercles sont proportionnels aux densités détectées pendant chaque séquence et sont centrés sur le milieu de la séquence. 
Cette représentation permet d'avoir un aperçu de la répartition des densités de poissons dans le milieu. On se rend compte d'une certaine hétérogénéité plus ou moins marquée seIon la période d'étude.

En janvier (fig. 6), l'hétérogénéité porte sur une différence entre les anses, plus fortement colonisées, et les grands axes profonds où les densités sont faibles $(<5$ poissons/ $1000 \mathrm{~m}^{3}$ ).

En mars (fig. 7), on observe une distribution très hétérogène avec des zones de fortes concentrations et des zones de faibles concentrations. II semble que se dessine plus nettement qu'en janvier un gradient de ré- partition zone littorale - zone pélagique, plus ou moins marqué selon les secteurs du lac: fortes concentrations en zones littorales, où sont le plus souvent détectés les bancs, faibles concentrations en zones pélagiques.

En juin (fig. 8), deux zones de fortes concentrations sont observées au centre et en queue de retenue. Le reste de la masse d'eau est colonisée de façon plus ou moins homogène par des densités de l'ordre de 75 à 150 poissons par $1000 \mathrm{~m}^{3}$.

\section{Estimation du nombre de poissons}

L'évaluation du nombre de poissons détectés par la méthode acoustique a

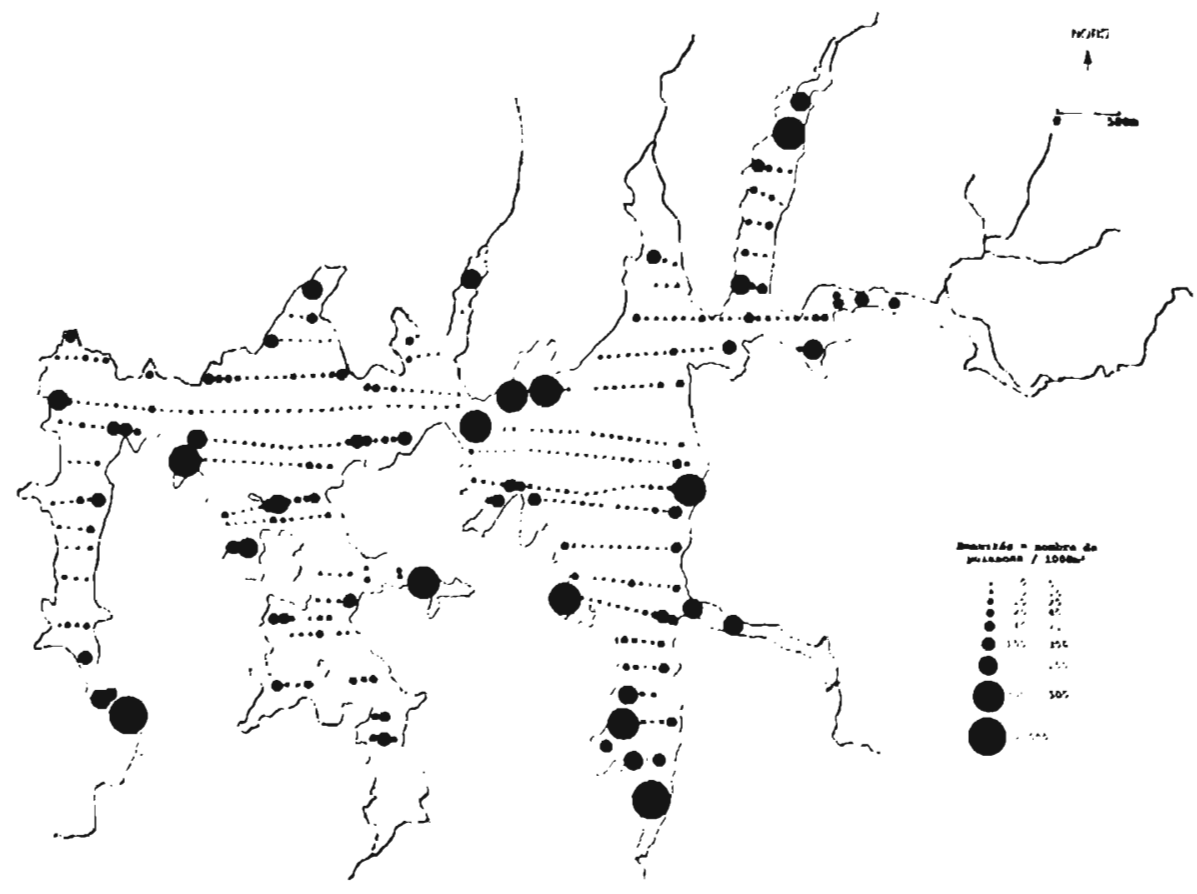

Fig. 6. - Carte de répartition des poissons en janvier 1993.

Fig. 6. - Map of fish distribution in January 1993. 


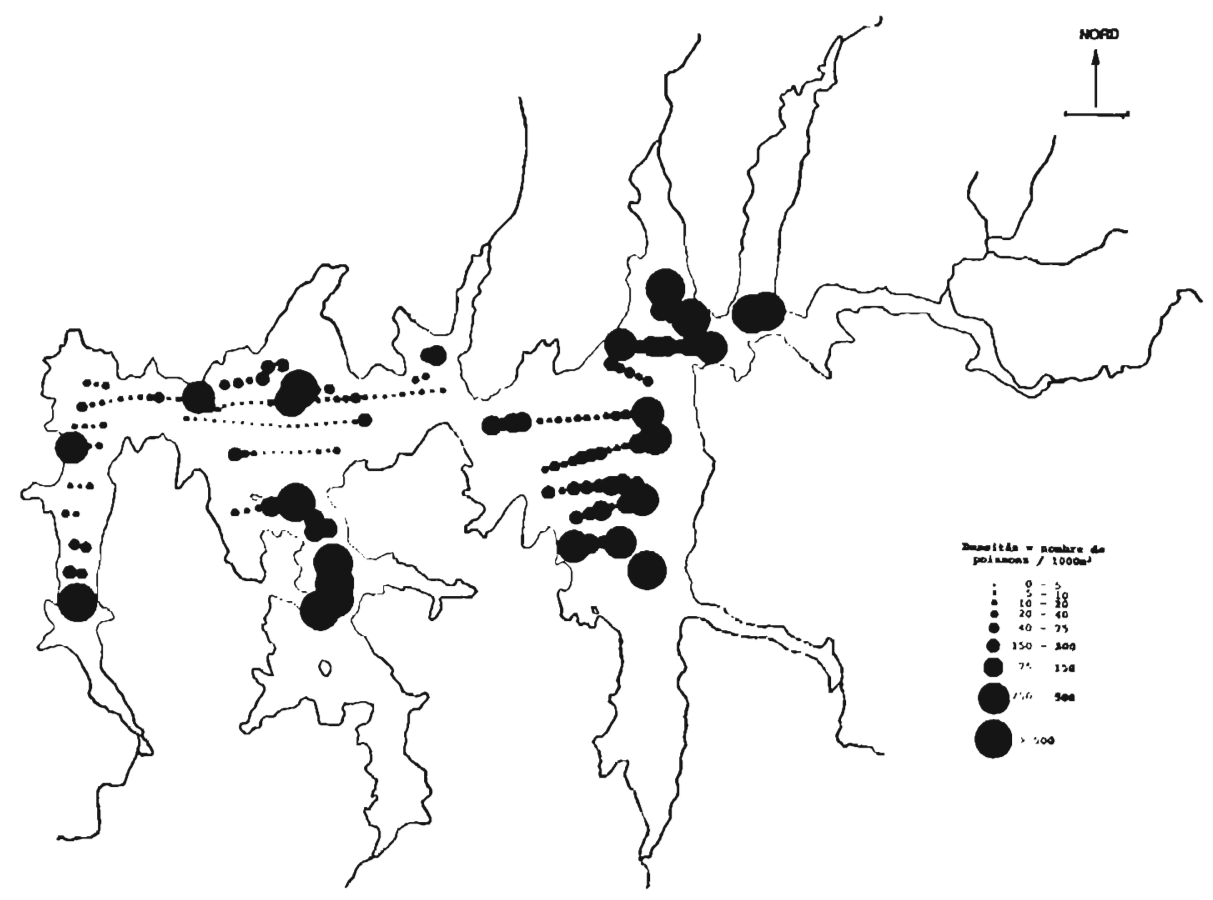

Fig. 7. - Carte de répartition des poissons en mars 1993.

Fig. 7. - Map of fish distribution in March 1993.

été obtenue par détermination du nombre de poissons par couche de $2 \mathrm{~m}$. Les résultats sont consignés dans le tableaul.

Le nombre total de poissons est donc $Y=\Sigma(y h * N h)=969050 \pm$ 255166. Des problèmes relatifs à l'évaluation de l'énergie renvoyée par les poissons (Cf. discussion $\S 3$ ) n'ont pas permis d'établir des histogrammes de fréquences d'énergies renvoyées par des échos isolés et de les comparer avec les histogrammes de fréquences de taille des poissons pêchés aux filets maillants lors de la campagne. II n'a donc pas été possi- ble d'obtenir un résultat fiable de l'estimation de la biomasse.

\section{Bilan de la vidange}

\section{Stock au niveau de la pêcherie}

Les étalonnages effectués les 30 premières heures ont consistés en une mesure individuelle de 17452 gardons, 2414 perches, 630 brèmes, 347 grémilles, plusieurs dizaines d'ablettes et autres espèces.

Un poids a été estimé pour chaque poubelle comptée en fonction de son 


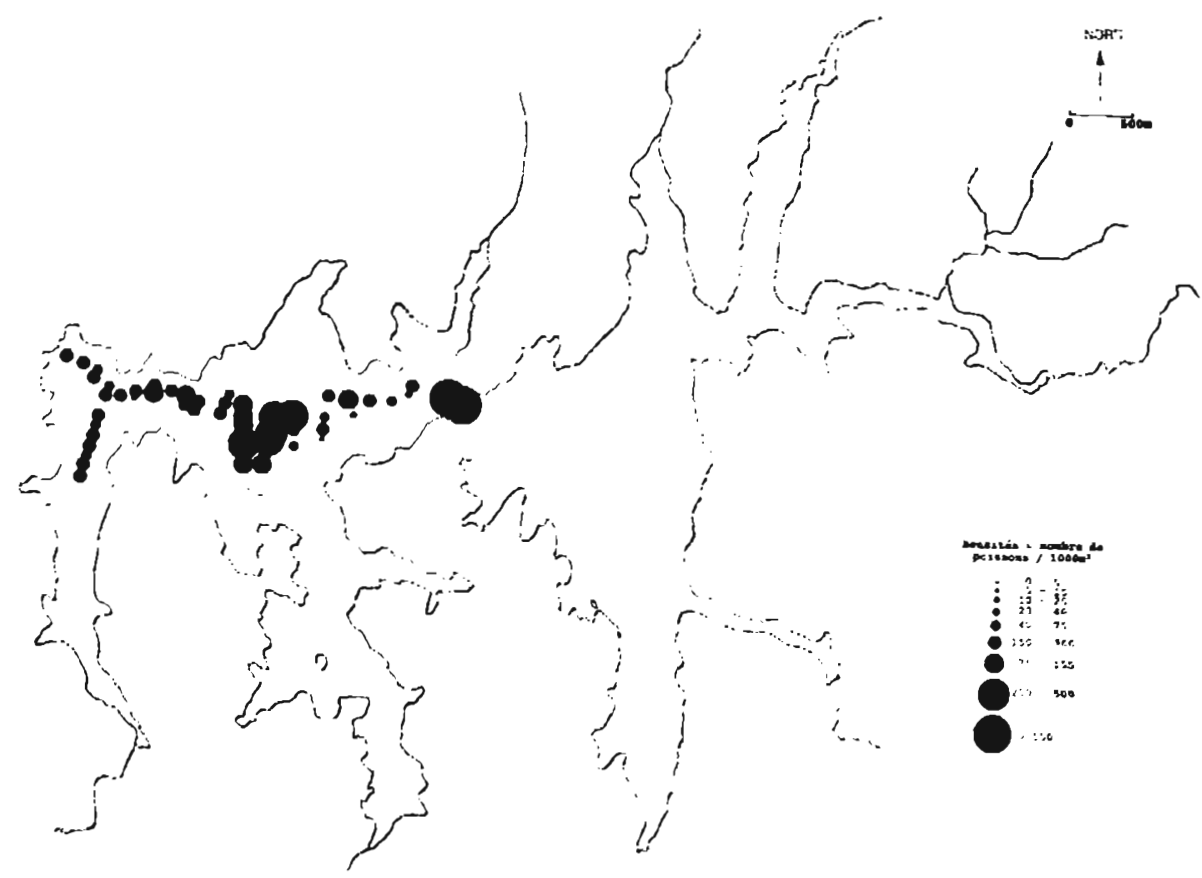

Fig. 8. - Carte de répartition des poissons en juin 1993.

Fig. 8. - Map of fish distribution in June 1993.

Tableau I. - Paramètres d'estimation de la population ichtyologique du lac de Pareloup. Table I. - Parameters for estimation of the ichthyological population of Pareloup.

\begin{tabular}{|c|c|c|c|c|c|c|}
\hline Strates & $h(m)$ & $0-2$ & $2-4$ & 4-6 & $6-8$ & $8-10$ \\
\hline Nombre d'échantillons & $\mathrm{nh}$ & 19 & 19 & 18 & 14 & 10 \\
\hline Densité (Nbre poissons $/ \mathrm{m}^{3}$ ) & $y h$ & 0,272 & 0,148 & 0,149 & 0,045 & 0,039 \\
\hline Ecart-type & Syh & 0,230 & 0,089 & 0,216 & 0,026 & 0,031 \\
\hline $\begin{array}{l}\text { Volume de la strate } h \\
\left(10^{3} \mathrm{~m}^{3}\right)\end{array}$ & $\mathrm{Nh}$ & 2188 & 1460 & 902 & 407 & 133 \\
\hline $\begin{array}{l}\text { Nombre de poissons par } \\
\text { strate yh }{ }^{*} \mathrm{Nh}\end{array}$ & & 595136 & 216080 & 134398 & 18315 & 5121 \\
\hline
\end{tabular}

niveau de remplissage (une poubelle pleine représentait environ $60 \mathrm{~kg}$ ).

Les résultats obtenus sont consignés dans le tableau II.
Avec plus de 530000 individus, le gardon est l'espèce la plus représentée. Puis viennent par ordre d'abondance: les perches (près de 64000 individus), les brèmes (plus de 
Tableau II. - Evaluation du stock des principales espèces présentes dans la retenue de Pareloup.

* Effectif et biomasse après extrapolation aux $38 \mathrm{~km}$ de ruisseaux du lac.

Table II. - Estimation of the stock of the main species present in Pareloup.

* Numbers and biomass after extrapolation to the $38 \mathrm{~km}$ of streams in the lake.

\begin{tabular}{|c|c|c|c|c|c|c|c|c|c|c|c|c|c|}
\hline & & Pèch & srie & Can & & Ruisse & eaux & Explo & tation & Echou & ués & Tot & \\
\hline Espèces & & Effectif & Poids & Effectif & Poids & EHectil & Poids & Effectif & Poids & Effectif & Poids & Effectif & Poids \\
\hline Ablettes & Alburnus alburnus (L.) & 281 & 15,5 & 0 & 0 & 0 & $\mathbf{0}$ & 23 & 0.9 & ? & $?$ & 304 & 16,4 \\
\hline Brèmes & Abramis brama (L.) & 30404 & 27060 & 4160 & 4580 & 0 & 0 & 479 & 429 & 150 & 130 & 35193 & 32199 \\
\hline Brochets & Esox lucius L. & 4682 & 1200 & 156 & 31 & 259 & 46,7 & 1256 & 3636 & $?$ & $?$ & 6353 & 4913,7 \\
\hline Carpes & Cyprinus carpio L. & 182 & 1460 & 0 & 0 & 0 & 0 & 51 & 458 & $?$ & $?$ & 233 & 1918 \\
\hline Chevesnes & Leucicus cephalus (L.) & 2 & 3,7 & 0 & 0 & 218 & 75,3 & 3 & 5 & $?$ & $?$ & 223 & 84 \\
\hline Gardons & Rutilus rutilus (L.) & 532315 & 30877 & 120677 & 7840 & 4518 & 365,5 & 147 & 8 & 350 & 800 & 671007 & 39890,5 \\
\hline Gol & Gobio gobio (L.) & 0 & 0 & 0 & 0 & 258 & 4,6 & $\mathbf{0}$ & 0 & $?$ & $?$ & 258 & 4,6 \\
\hline Gremilles & Gymnocephalus cernua (L.) & 5976 & 39,2 & 27 & 0,2 & 0 & 0 & 0 & 0 & $?$ & $?$ & 6003 & 39,4 \\
\hline Loches & Nemacheilus barbatulus (L.) & 0 & 0 & 0 & 0 & 13 & 0,06 & 0 & 0 & $?$ & $?$ & 13 & 0.06 \\
\hline Perches & Perca fluviatilis L. & 63632 & 4323 & 2933 & 222 & 1609 & 90,5 & 45 & 2 & $?$ & $?$ & 68219 & 4637,5 \\
\hline Sandres & Stizostedion fucioperca (L.) & 427 & 150 & 73 & 25 & 0 & 0 & 1290 & 4448 & ? & $?$ & 1790 & 4623 \\
\hline $\operatorname{Tan}$ & Tinc & 7 & 74 & 4 & 4 & 0 & 0 & 0 & 0 & 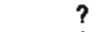 & $?$ & 80 & 78 \\
\hline Truite & Salmo trutta fario $\mathrm{L}$. & 15 & 10 & 0 & 0 & 1444 & 168,6 & 60 & 250 & $?$ & $?$ & 1519 & 428,6 \\
\hline Vairons & Phoxinus phoxinus (L.) & 40 & 0,5 & 27 & 0,1 & 0 & 0 & 0 & 0 & $?$ & $?$ & 67 & 0,6 \\
\hline Vandoises & Leuciscus leucicus (L.) & 7 & 13,4 & 81 & 13,7 & 114 & 10,6 & 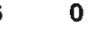 & 0 & 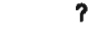 & $?$ & 272 & 37,8 \\
\hline Alevins dén & mbrés & N.D. & N.D. & 66862 & 334 & N.D. & N.D. & 0 & 0 & $?$ & $?$ & N.D. & N.D. \\
\hline Total & dultes & 638109 & 65226 & 128138 & 12716 & $8433^{\circ}$ & $762^{*}$ & 3354 & 9237 & 13500 & 930 & 791534 & 88871 \\
\hline
\end{tabular}



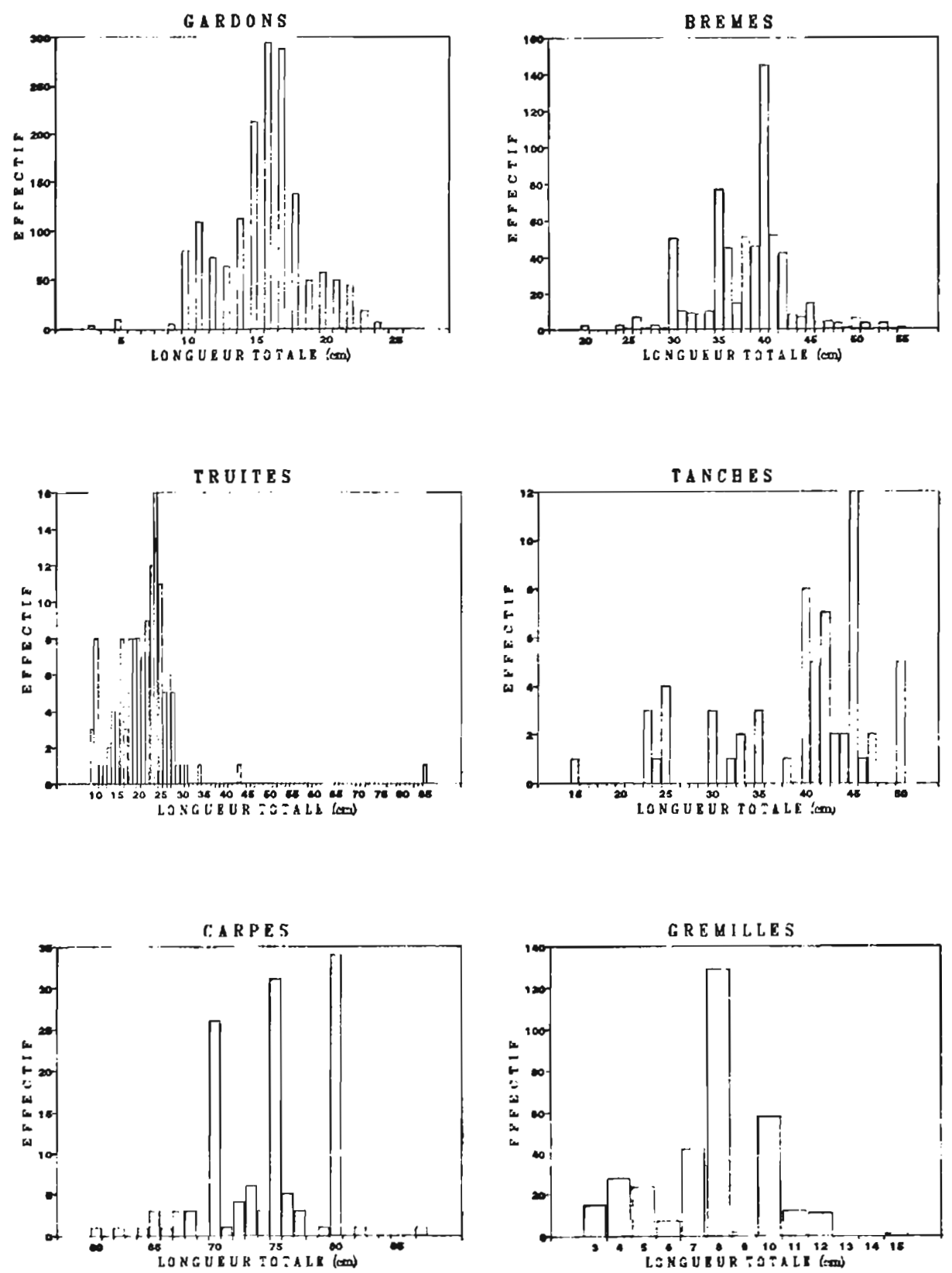

Fig. 9. - Histogrammes des classes de taille des principales especes.

Fig. 9. - Size-class histograms of main species. 

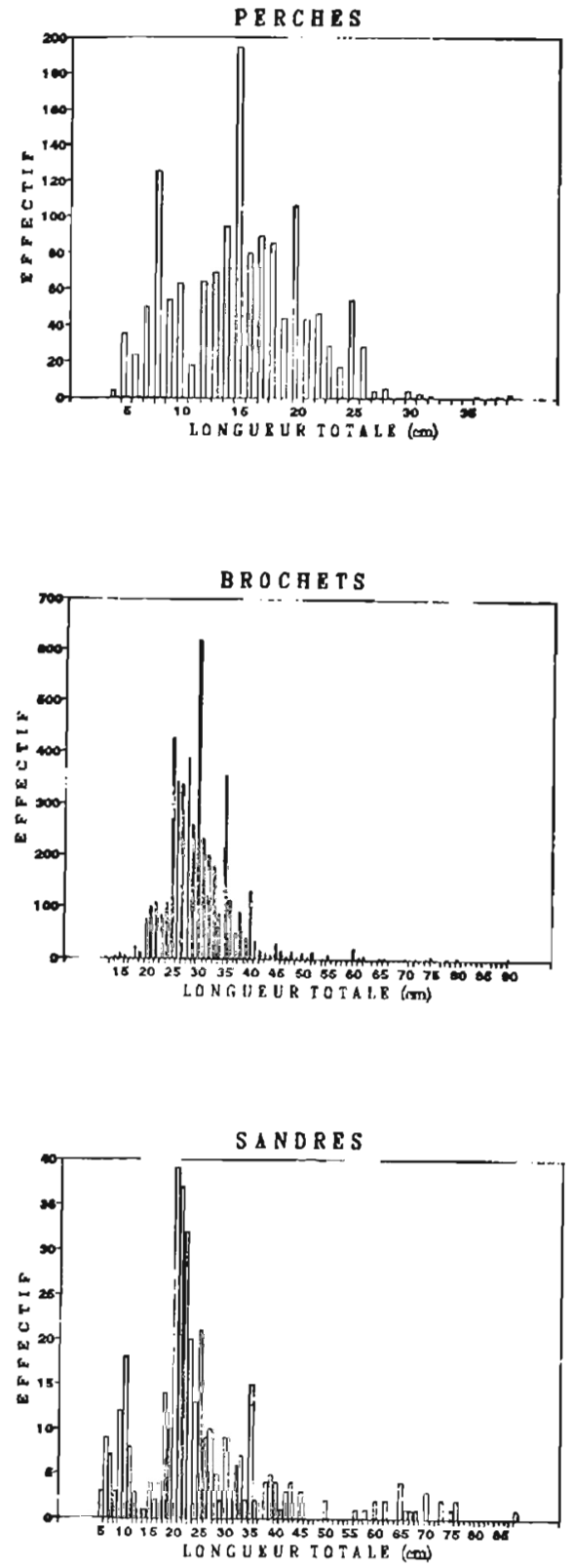

Fig. 9. - Suite

Fig. 9. - Continued
30000 ) et, en quantité moindre, les grémilles, brochets, sandres, ablettes, carpes, tanches, etc.

L'ordre d'importance pondérale de ces espèces est différent : si les gardons dominent avec 31 tonnes, les brèmes approchent ce tonnage (27 T.) ; viennent ensuite par ordre décroissant: les perches $(\approx 4,6 \mathrm{~T}$.), les carpes $\quad(\approx 1,5 \mathrm{~T}$.), les brochets $(\approx 1,2 \mathrm{~T}$.), les autres espèces étant beaucoup plus faiblement représentées $(<1 \mathrm{~T}$.).

La répartition par classe de taille des individus appartenant aux principales espèces a été établie (fig. 9) d'après les mesures effectuées lors de l'étalonnage, à l'exception des brochets, sandres, carpes, tanches et truites qui ont tous été mesurès, voire pesés.

Ces histogrammes mettent en évidence une absence d'individus de petite taille. La majorité des poissons inférieurs à $6 / 7 \mathrm{~cm}$ s'est en effet réfugiée dans le canal, est passée à travers les grilles de la pêcherie, ou est restée au niveau des ruisseaux et a ainsi échappé au recensement.

\section{Stock au niveau des ruisseaux}

Au total, 19 pêches électriques ont été effectuées sur l'ensemble des principaux ruisseaux (fig. 5). Chaque secteur de ruisseau est représenté par une ou deux stations "caractéristiques" regroupant l'ensemble des caractères rencontrés sur le secteur (largeur, profondeur, débit, nature du fond, variété des caches...). La quantité (nombre et biomasse) de poissons 
pêchés dans ces stations a été extrapolée aux secteurs qu'elles représentent. Les résultats de ces pêches sont consignés dans le tableau II et fig. 10.

Le gardon est l'espèce la plus abondante avec la perche; un nombre important de truites, chevesnes et goujons a été capturé, espèces peu représentées au niveau de la pêcherie.

On note également une capture de plusieurs milliers d'alevins de gardons et/ou de chevesnes et de vandoises (stations 3, 7, 8, 10).

Le Connes s'est révélé être le ruisseau le plus riche (station 4: 3778 poissons/ha, truites et gardons dominants), suivi du Vioulou amont (station 10: 2500 poissons/ha, perches et gardons dominants; station $9: 1943$ poissons/ha, gardons et perches dominants en nombre, chevesnes dominants en biomasse). Le Roucan (station 2: 2133 poissons/ha, truites dominantes) et le Rieutord (station 1 : 2143 poissons/ha, gardons dominants et station $3: 2333$ poissons/ha, gardons et perches dominants) se sont avérés être également fortement peuplés.

En revanche, les ruisseaux temporaires ou à faible débit et faible profondeur ne renfermaient que peu (stations $7,13,15$ ) ou pas (stations $16,17,18,19)$ de poissons ou n'étaient colonisés que par des alevins (station 8).
Finalement, nous avons estimé à près de 8500 , le nombre de poissons adultes restés au niveau des différents ruisseaux pour une biomasse d'environ $760 \mathrm{~kg}$.

\section{Stock au niveau de la conduite forcée}

Plus de 120000 gardons $(\approx 7,8 \mathrm{~T}$ ) , 4160 brèmes $(\approx 4,6 \mathrm{~T}$.), plus de 2900 perches $(\approx 0,2 \mathrm{~T}$.), 156 brochetons, 81 vandoises, 73 sandres et quelques représentant d'autres espèces ont été extrait de la galerie reliant le lac de Pareloup au lac de Villefranche de Panat (Tableau II).

Nous avons également estimé à près de 67000 le nombre d'alevins réfugiés dans le canal, soit avec les adultes, une biomasse totale avoisinant les $13 \mathrm{~T}$.

\section{Nombre de poissons échoués}

La majorité des poissons morts se sont échoués au pied du barrage sur une surface d'environ $2700 \mathrm{~m}^{2}(15 \mathrm{~m}$ $\times 180 \mathrm{~m})$. La densité de poissons échoués a été évaluée à 5 par $\mathrm{m}^{2}$, soit un total de 13500 individus majoritairement constitués de gardons.

Quelques cadavres ont été retrouvés entre les vannes du barrage et la pêcherie, mais en quantité négligeable.

Fig. 10. - Nombre (à gauche) et biomasse en g. (à droite) des poissons capturés par pêches électriques.

Fig. 10. - Number (on left) and biomass in g. (on right) of fish captured by electrofishing. 

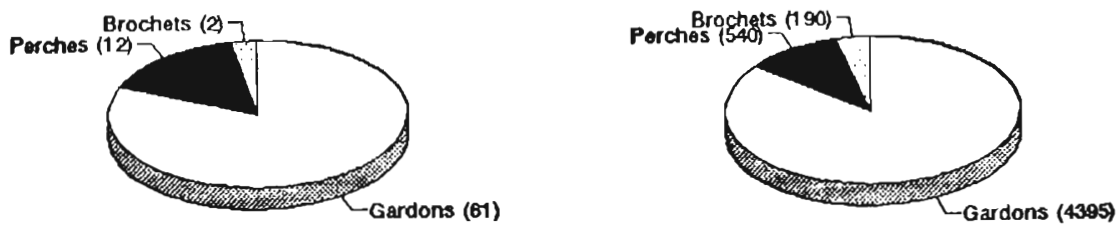

STATION 2

Surface pachée: $150 \mathrm{~m}^{2}$
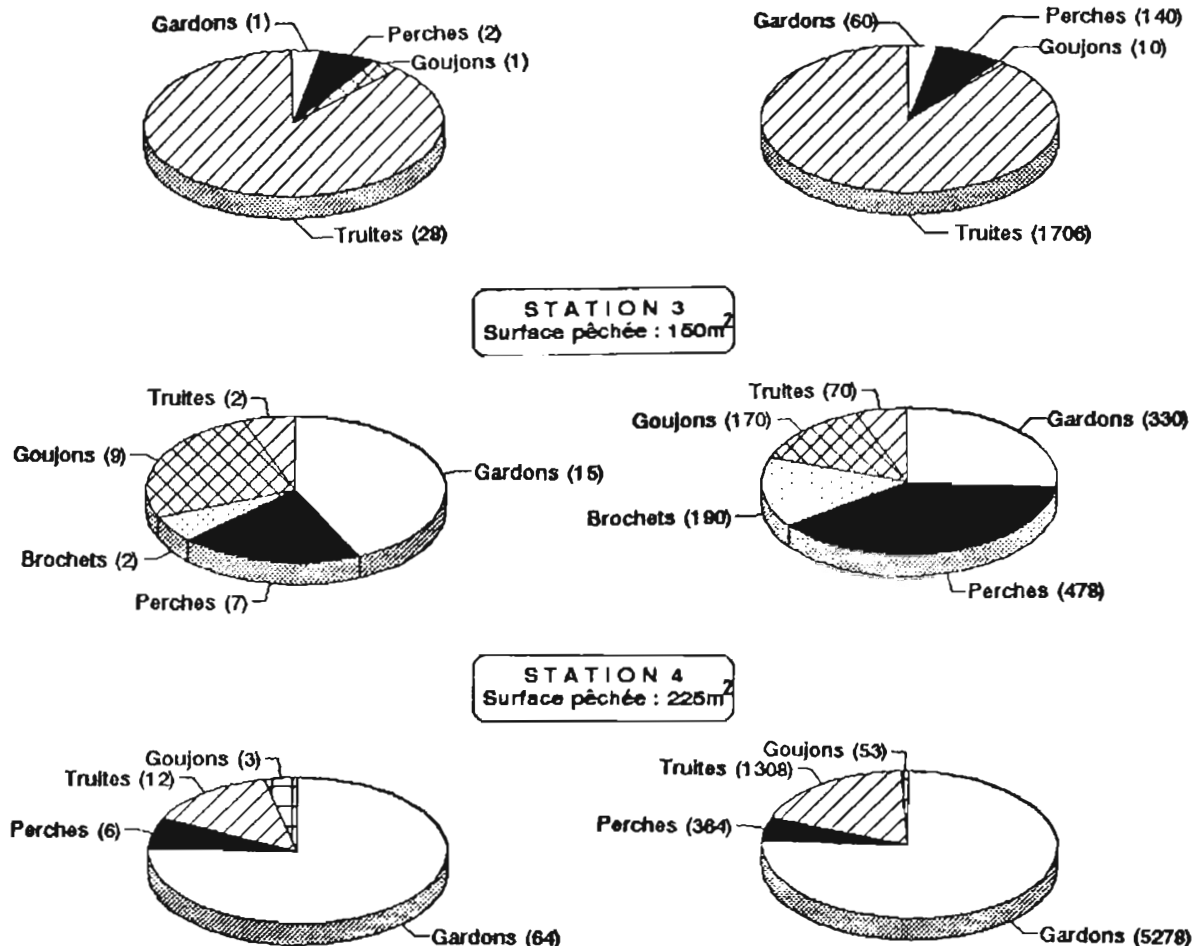

Surface péchée : $220 \mathrm{~m}^{2}$
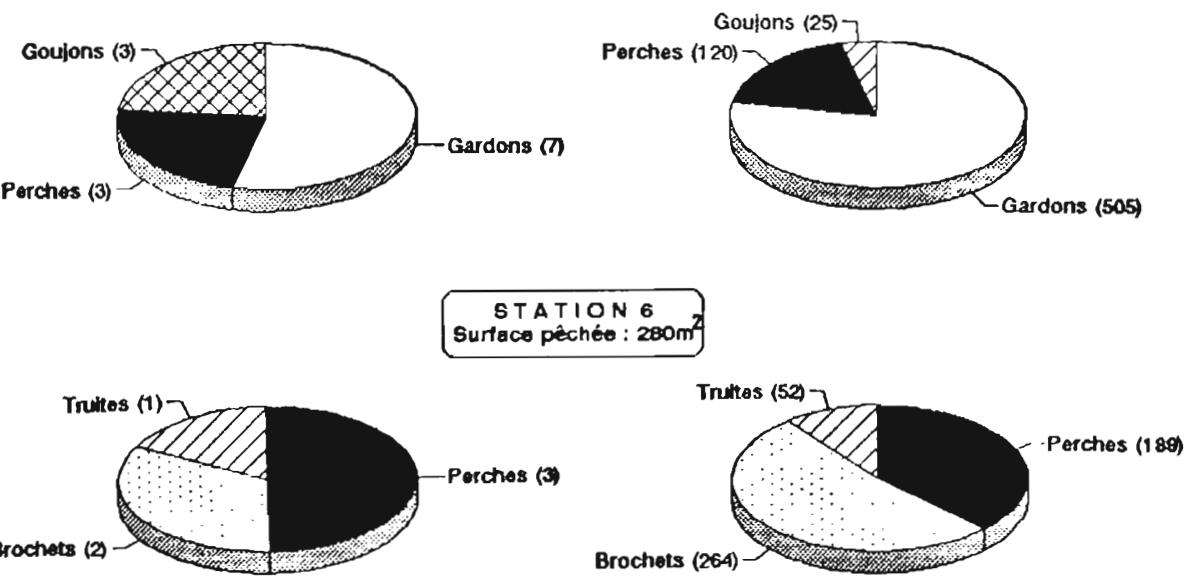

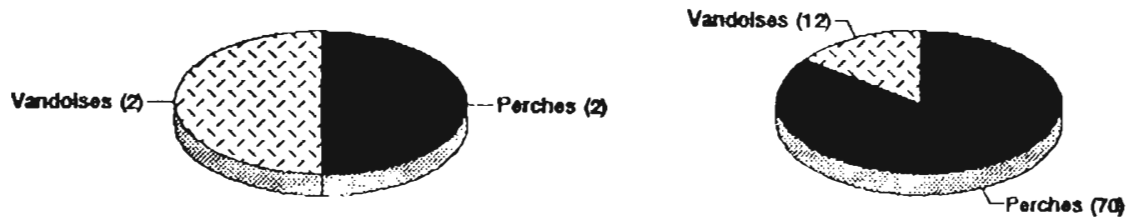

STATION

Surface pechéo : $700 \mathrm{~m}^{2}$
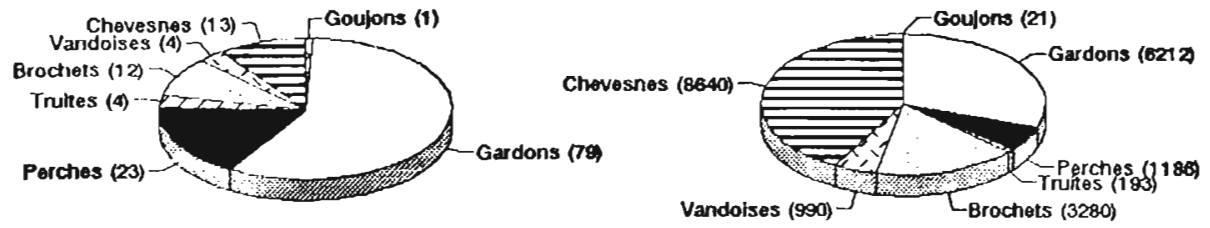

STATION 10

Surtace pechbe: $1000 \mathrm{~m}^{2}$
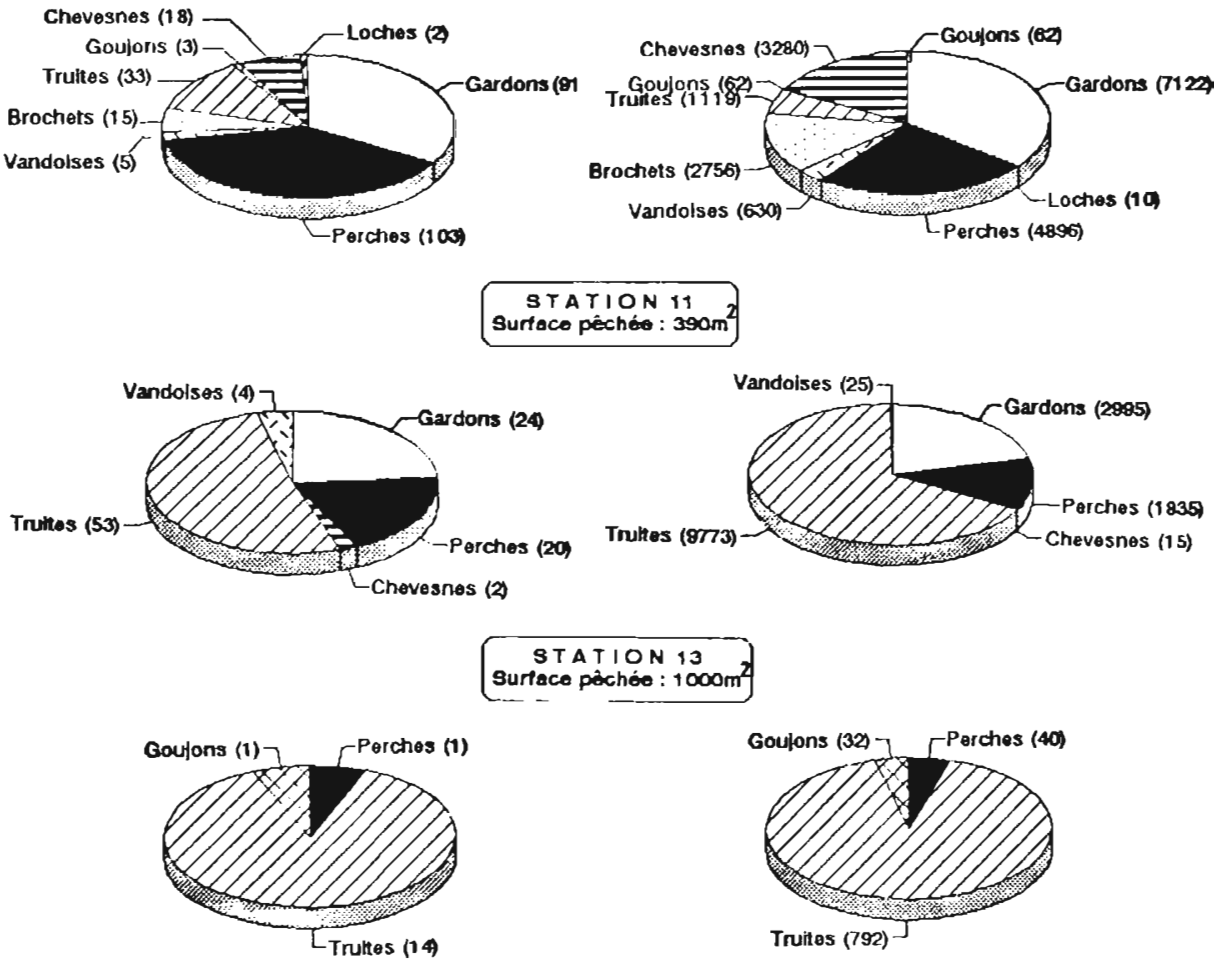

STATION 13

Surface paché : $1000 \mathrm{~m}^{2}$
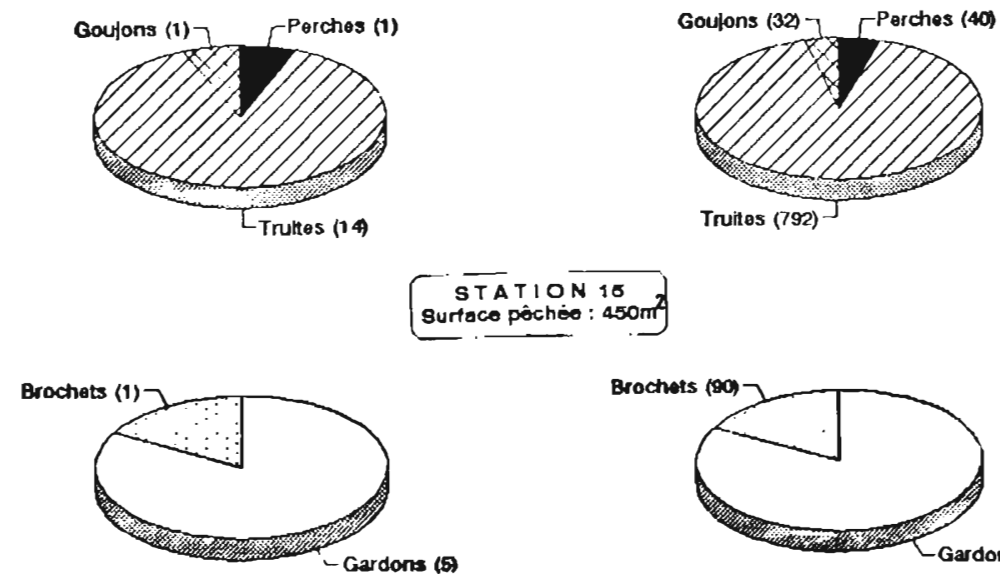

STATION 10

urtace pôcheo : 450

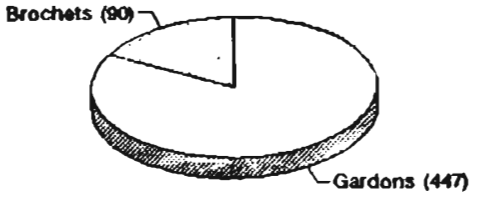


L'estimation du nombre de poissons échoués est approximative et ne représente qu'un ordre de grandeur.

\section{Nombre de poissons pêchés lors de la vidange}

Les résultats de l'enquête publique, auxquels il convient d'ajouter les résultats d'une campagne de pêche au filet la semaine précédant la vidange, font apparaître une prise importante de carnassiers: 1256 brochets $(\approx 3,6 \mathrm{~T}$. $)$ et 1290 sandres $(\approx 4,5 \mathrm{~T}$. ont été retirés du lac (tableau II).

\section{Bilan de l'inventaire}

Le nombre total de poissons adultes a finalement été évalué à plus de 790000 pour une biomasse totale d'environ 89 tonnes, soit plus de $68 \mathrm{~kg} / \mathrm{ha}$ lorsque le lac est à sa cote maximale.

\section{DISCUSSION}

\section{Comportement des poissons}

Quelques remarques peuvent être formulées concernant le déroulement de la vidange et le comportement de certaines espèces.

L'arrivage des poissons au niveau de la pêcherie a été progressif: les premières espèces recueillies ont été essentiellement les gardons, les perches et les grémilles. Le suivi des mouvements des poissons par échosondage lors de la baisse de niveau a montré que ceux-ci restaient en queue de retenue traduisant un caractère rhéophile, ce qui laissait prévoir un arrivage peu abondant en début de vidange. Puis, le flux de dévalaison s'est accentué à partir du second jour révélant des quantités importantes de brèmes. D'après les observations de Lim et al. (1990) lors de la vidange de la retenue des Saints-Peyres, le flux de dévalaison peut être initié par la variation de la qualité physico-chimique de l'eau, notamment des teneurs en M.E.S., en oxygène dissous et en $\mathrm{N} / \mathrm{NH}^{+}$.

Difficile au vu du bilan physico-chimique (Andrieu, 1993) d'établir une corrélation directe entre flux de dévalaison des poissons et caractéristiques physico-chimiques de l'eau; si les variations de ces caractéristiques ont pu jouer un rôle, il n'est pas forcément prépondérant à Pareloup. En revanche, il semble plus probable que l'augmentation du flux de dévalaison soit conditionné par une diminution de l'espace vital des poissons ce qui corroborerait les observations de Lim et al. (op. cit.) qui suggèrent l'existence d'un "espace vital critique" au dessous duquel les poissons cesseraient de lutter contre la dévalaison.

La fin de la vidange a été marquée par la récupération des poissons fouisseurs (carpes et tanches) entraînés avec le fond du culot.

Certaines espèces, en revanche, n'ont pas (goujons, loches) ou peu (chevesnes, truites, vairons) été recensées au niveau de la pêcherie.

La faible représentation des chevesnes et des truites traduit un 
comportement particulièrement rhéophile de ces deux espèces qui ont réussi à échapper à la vidange en remontant les rivières. Cette migration a été mise en évidence grâce aux pêches électriques qui ont montré des quantités importantes de truites dans le Roucan et le Vioulou, et de chevesnes surtout dans le Vioulou.

L'absence de goujons et de loches, et le peu de vairons peuvent s'expliquer par leur petite taille qui leur aurait permis de passer à travers les grilles de la pêcherie au même titre qu'une majorité d'alevins, et ainsi échapper au recensement. Les pêches électriques effectuées aux stations $3,4,5,9,10$ et 13 montrent en effet que ces espèces sont habituellement présentes dans l'écosystème.

Les poissons récupérés en début de vidange étaient en meilleur état que ceux récupérés vers la fin après avoir séjourné dans les eaux troublées par la remise en suspension des sédiments. Cette remise en suspension s'est traduite par une augmentation brutale de la turbidité de l'eau à la sortie des vannes le 16 juin à 3 heures, le taux de M.E.S. passant alors de $0,09 \mathrm{~g} / \mathrm{l}$ a $1,93 \mathrm{~g} / \mathrm{l}$, pour augmenter régulièrement et rapidement les 2 heures suivantes (Andrieu, 1993).

Le brochet, vraisemblablement en raison de sa forme longiligne, semble être l'espèce ayant le moins souffert de la dévalaison, même en fin de vidange; la quasi totalité des brochets a ainsi pu être sauvée. Une proportion importante de brochetons de 25 à
$30 \mathrm{~cm}$ (fig. 9) dénote des conditions de reproduction favorables en 1991 . 92. Quelques gros individus ne figurant pas sur la figure 9 ont également été récupérés : 6 brochets de $100 \mathrm{~cm}$, 2 de $103 \mathrm{~cm}, 2$ de $113 \mathrm{~cm}, 2$ de $120 \mathrm{~cm}, 2$ de $121 \mathrm{~cm}$ et un de $132 \mathrm{~cm}$.

Soulignons enfin que l'absence de carpes de longueur inférieure à $60 \mathrm{~cm}$ (fig. 9) pourrait démontrer la stérilité de l'espèce sans doute en raison de la température moyenne trop basse à Pareloup et/ou de la vieillesse du cheptel qui se renouvelle mal (prédation, marnage).

\section{Composition du peuplement}

La composition du peuplement montre que Pareloup au moment de la vidange est une retenue cyprinicole abritant 15 espèces différentes largement dominées par le gardon (Rutilus rutilus (L.)) et la brème (Abramis brama (L.)), ces deux espèces représentant à elles seules plus de $89 \%$ du nombre et plus de $80 \%$ de la biomasse totale.

Les pêches au filet réalisées jusqu'à présent à Pareloup (Dauba \& Tourenq, 1983; Béarez, 1988) avaient bien mis en évidence un développement très important des populations de brèmes ces dernières années. Lors de l'estimation faite en 1983, les brèmes ne contribuaient que pour $13 \%$ à la biomasse totale, elles représentent aujourd'hui près de $36 \%$. On constate également une contribution pondérale dominée par les gar- 
dons : plus de $44 \%$ contre seulement $22 \%$ en 1983.

Les deux espèces représentaient en 1983 près de $60 \%$ en nombre et plus de $35 \%$ en biomasse; en 1988 , ces chiffres passaient à $71 \%$ pour le nombre et près de $73 \%$ pour la biomasse.

Cet accroissement important de la productivité de brèmes et de gardons traduit un vieillissement de l'écosystème (Hartmann, 1977, 1979).

Notons enfin que les trois carnassiers présents, le sont dans des proportions comparables: brochet (Esox Iucius L.), 4,9 T., sandre (Stizostedion lucioperca (L.)), 4,6 T. et perche (Perca fluviatilis L.), 4,6 T., aux erreurs d'étalonnage près (étalonnage des poubelles discutable).

\section{Comparaison avec les estimations acoustiques. Problèmes rencontrés}

\section{Nous avions estimé à environ} 969000 , le nombre de poissons détectés par échosondage en juin, soit une surestimation d'environ 177000 $(+22,4 \%)$ par rapport au bilan global de la vidange. Une explication rationnelle de cette surestimation réside dans les problèmes d'estimation des quantités de poissons envasés et passant par les grilles de l'exutoire (Degiogi et al., 1993).

Les trois campagnes réalisées ont permis de mettre en évidence les lacunes de l'échosondeur utilisé en matière de T.V.G. (Time Varied Gain). Ce dispositif, inclus dans l'échosondeur, est chargé de compenser les pertes par étalement géométrique du faisceau conique émis. Ses spécificités inadéquates ont conduit à une augmentation du bruit acoustique en surface et ont limité la possibilité d'obtenir des résultats quantitatifs fiables par la suite. Les pertes ont été minimes en juin en raison de la faible profondeur moyenne du lac.

Malheureusement, si le nombre de poissons estimé paraît correct, il nous a été impossible de travailler sur l'énergie renvoyée. Impossible donc dans ces conditions d'établir de comparaisons entre fréquences de tailles des poissons capturés au filet et fréquence d'énergies renvoyées par des échos isolés. Impossible donc d'établir la proportion des individus de petite taille par rapport aux gros et de déterminer ainsi la biomasse totale sans erreur importante.

Les cartes de répartition (fig. 6, 7 , 8 ) ayant permis de suivre le mouvement des poissons lors de la baisse de niveau, demeurent néanmoins correctes. En outre, les erreurs sont uniformément réparties et la répartition des poissons dans le plan horizontal est conforme aux observations faites dans d'autres écosystèmes (Guillard, 1991).

Cette expérience nous a permis d'apporter les corrections et modifications nécessaires et indispensables au bon fonctionnement de l'appareillage acoustique. Elle a également été l'occasion de vérifier les performances du système de localisation par satellites (GPS) en mode différentiel qui 
nous a permis de reconstituer sur des cartes le parcours exact du zodiac.

\section{CONCLUSION}

La vidange de la retenue hydroélectrique de Pareloup a permis de dresser un inventaire relativement complet de sa faune piscicole. Avec plus de 790000 poissons adultes pour une biomasse de l'ordre de 89 tonnes, cette retenue cyprinicole dont le peuplement est dominé par les gardons et les brèmes, s'est avéré relativement riche: $68 \mathrm{~kg} / \mathrm{ha}$ (Cote $805 \mathrm{NGF}$ = cote maxi).

Ces chiffres ne tiennent pas compte de la dizaine de tonnes (dont $2,4 \mathrm{~T}$. de poissons nobles : brochets, sandres, perches et truites) extraite du lac entre le $1^{\mathrm{er}}$ janvier et le $1^{\mathrm{er}}$ juin 1993 dans le cadre de pêches scientifiques réalisées par I'U.P.S. et l'E.N.S.A.T., de pêches effectuées par I'A.P.P. de Rodez ou par les pêcheurs autochtones.

Dans le cadre de la mise au point d'un système d'acquisition et de traitement des échos robuste, léger, transportable sur un zodiac et performant, la vidange a été l'occasion de vérifier la fiabilité du matériẹ par comparaison des résultats acoustiques et du bilan de la vidange. Les problèmes rencontrés au niveau de la fonction T.V.G. de l'échosondeur, dont les signaux étaient issus, n'ont pas permis une étude quantitative précise.

Une modification et un étalonnage précis ont permis un échantillonnage correct des lacs de Bage et Pont de Salars (Aveyron) par une amélioration de la dynamique du système et la mise en place d'une fonction T.V.G. performante.

Deux facteurs permettront l'obtention de résultats précis: la connaissance exacte des volumes par strate et l'application d'un traitement statistique approprié. L'élaboration d'un modèle est actuellement en cours au Laboratoire de Statistiques et de Probabilités de I'U.P.S., modèle permettant l'obtention d'un intervalle de confiance de l'estimation beaucoup plus étroit que celui présenté dans la méthode utilisée ici.

Cette vidange a permis, en outre, de tirer certains enseignements: une meilleure concertation "pré-vidange" s'impose de manière à mieux gérer l'arrivée massive des poissons, à mieux ordonner la collecte des données et à augmenter les étalonnages de contrôle (vérification des quantités et proportions des espèces par poubelle) pour des résultats plus précis.

Fort de cette expérience et de l'amélioration du matériel de détection acoustique, nous devrions aborder la vidange des lacs de Bage (septembre 1994) et de Pont de Salars (automne 1995) dans les meilleures conditions.

Nous pourrons également suivre la recolonisation (zones de répartition, contrôle de la reproduction) du lac de Pareloup par les poissons restés au niveau des ruisseaux et de la retenue artificielle (fig. 5). Ce stock, essentiellement constitué de gardons $(48 \%$ de la biomasse), de truites (22\%), de 
perches $(12 \%)$ et de chevesnes $(10 \%)$ et estimé à 1 tonne sera enrichi par d'autres individus par la Fédération et les Associations de pêche de l'Aveyron.

\section{REMERCIEMENTS}

Les auteurs tiennent à exprimer leurs sincères remerciements à tous ceux qui ont participés activement au fastidieux travail de récupération, de tri et de mensuration des poissons lors de la vidange du lac de Pareloup: membres et responsables des Association de Pêche du département de l'Aveyron, chercheurs et étudiants du Laboratoire d'Hydrobiologie de l'Université Paul Sabatier.

Le dévouement et la constance de Melles C. Mur et V. Sarpedonti ont été particulièrement appréciés. Que soient également remerciés Mr. A. Mayet, technicien au L.A.M.I., pour sa disponibilité sur le terrain lors des campagnes d'échosondage, Melle F. Sivadier et Mr. J. Dagnac pour leur aide lors des pêches électriques, ainsi que Mr. J.M. Thébault qui a réalisé la majorité des photos qui illustrent cet article.

Nos remerciements s'adressent enfin à tous les responsables et personnels des divers services d'Electricité de France qui ont toujours facilité notre travail.

\section{RÉFÉRENCES}

Andrieu J.P., 1993. Résultats du suivi des principaux parametres physico-chimiques. Vidange de la retenue de Pareloup sur le Vioulou. Juin 1993. Rapport EDF/DTG-RE/VID0054A.

Bazigos G.P., 1975. The statistical efficiency of echo surveys with special reference to Lake Tanganyika. Rome, FAO, FIPST/T 139, $52 \mathrm{p}$.
Bagenal T.B., Dahm E., Lindem T. et Tuunainen P., 1982. EIFAC experiments on pelagic fish stock assessment by acoustic methods in Lake Konnevesi, Finland. FAO, EIFAC Occas. Pap., (14) : $16 \mathrm{pp}$.

Béarez P., 1988. Populations piscicoles du lac de Pareloup (Aveyron). Etude de la croissance. DEA d'Ecologie, UPS Toulouse, $53 \mathrm{p}$.

Blaxter J.H.S. et Batty R.S., 1990. Swimbladder "behaviour" and target strength. Rapp. P.-v. Réun. Cons. int. Explor. Mer, 189 : 233-244.

Burczynski J.J. et Karp W.A., 1985. The application of dualbeam acoustic survey techniques for fish population estimation in lakes. AFS Symp. on Small Hydropower and Fischeries, Denver, Colorado, May 1-3, $3 \mathrm{p}$.

Dahm E., Hartmann J., Lindem T. et Loffler H., 1985. EIFAC experiments on pelagic fish stock assessment by acoustic methods in Lake Constance. FAO, EIFAC Occas. Pap/Doc. Occas. CECPI, (15): $14 \mathrm{p}$.

Dauba F. et Tourenq J.N., 1983. Etude hydrobiologique du lac de Pareloup (Aveyron). E.N.S.A.T. - Rapp. EDF (REAM) : $64 \mathrm{p}$.

Degiorgi F., Guillard J., Grandmottet J.P. et Gerdeaux D., 1993. Les techniques d'étude de l'ichtyofaune lacustre utilisée en France : bilan et perspectives. Hydroécol. Appl. 5(2), pp. 27-42.

Foote K.G., Knutsen H.P., Vestnes G., Mac Lennan D.N. et Simmonds E.J., 1987. Calibration of acoustic instruments for fish density estimation: a practical guide. ICES Coop. Res. Rep. 144. $69 \mathrm{pp}$.

Gerlotto F., 1989. Choise of a strategy for sampling and stratifying the fish concentrations in tropical echo integration. In ICES-CM-1989/b:47. ICES (Copenhagen, Denmark), Council Meeting 1989 (Coll. Paper) : 24 pp.

Guillard J., 1991. Etude des stocks pisciaires lacustres par échointégration: 
problèmes méthodologiques. Thèse Doct., Université Claude Bernard, Lyon I, $156 \mathrm{p}$.

Hartmann J., 1977. Fische reiliche veränderungen in Kulturbedingt eutrophierenden Seen. Schweiz. Z. Hydrol., 39, 243-254.

Hartmann J., 1979. Unterschiedliche adaptionsfähigkeit der fische an eutrophierung. Schweiz. Z. Hydrol., 41(2), 374-382.

Hartmann J., Dahm E., Dawson J., Doering P., Jürgensen S., Lindem T., Löffler $H_{\text {., }}$ Raemhild G. et Völzke V., 1987. EIFAC experiments on pelagic fish stock assessment by acoustic methods in Lake Tegel. FAO, EIFAC Occas. Pap., (17): $26 \mathrm{p}$.

Jolly G.M. et Hampton I., 1990. Some problems in the statistical design and analysis of acoustic surveys to assess fish biomass. Rapp. P.-v. Réun. Cons. int. Explor. Mer, 189 : 415-420.

Jurvelius J., Lindem T. et Louhimo J., 1984. The number of pelagic fish in Lake Paasivesi, Finland, monitored by hydroacoustic methods. Fish. Res., 2 : 273-283.

Jurvelius J. et Heikkinen T., 1987. The pelagic fish density, biomass and growth of vendace, Coregonus albula L., monitored by hydroacoustic methods and trawling in a Finnish lake. Aqua Fennica, 17, 1:27-34.

Junvelius J., Lindem T. et Louhimo J., 1987. The number of fish in the pelagic areas of Lake Pyhajarvi (Karelia), monitored by hydroacoustic methods. Finnish Fish. Res., 8, 48-52.

Laloë F., 1985. Contribution à l'étude de la variance d'estimateurs de biomasse de poissons obtenus par échointéqration. Océanoqr. trop. 20 (2) : 161-169.

Lévénez J.J., Gerlotto F. et Petit D., 1990. Reaction of tropical coastal pelagic species to artificial lighting and implications for the assessment of abundance by echo integration. Rapp. P.-v.
Réun. Cons. int. Explor. Mer, 189 : 128-134.

Lim P., Sabaton C. et Belaud A., 1990. Inventaire piscicole de la retenue des Saints-Peyres (Tarn) à l'occasion d'une vidange décennale. Hydroécol. Appl. 1/2, pp. 5-19.

Marchal E. et Boely T., 1977. Evaluation acoustique des ressources en poissons du plateau continental ouest-africain des îles Bissagos $\left(11^{\circ} \mathrm{N}\right)$ à la pointe Stafford $\left(28^{\circ} \mathrm{N}\right)$. Cath. O.R.S.T.O.R.M., sér. Océanogr., vol. XV, n²2: 139-161.

Ponton D. et Meng H.J., 1990. Use of dual-beam acoustic technique for detecting young whitefish, Coregonus $s p .$, juveniles : first experiments in an enclosure. J. Fish. Biol. : 36, 741-750.

Rose G.A. et Leggett W.C., 1988. Hydroacoustic signal classification of fish schools by species. Can. J. Fish. Aquat. Sci. 45: 597-604.

Salvetat R., 1992. Application des techniques d'échosondage aux études limnologiques: apport des processeurs de traitement du signal. Thèse (Doct. d'Univ.), Université Paul Sabatier, Toulouse III, $126 \mathrm{p}$.

Thornes R.E., 1990. Session 5 : Rapporteur's review. Rapp. P.-v. Réun. Cons. int. Explor. Mer, 189: 192-196.

Traynor J.J., Williamson N.J. et Karp W.A., 1990. A consideration of the accuracy and precision of fish-abundance estimates derived from echo-integration surveys. Rapp. P.-v. Réun. Cons. int. Explor. Mer, 189 : 101-111.

Walline P.D., Pisanty S. et Lindem T., 1992. Acoustic assessment of pelagic fish in Lake Kinneret, Israel. Hydrobiologia 231: 153-163.

Williamson N.J., 1982. Cluster sampling estimation of the variance of abundance estimates derived from quantitative echo sounder surveys. Can. J. Fish. Aquat. Sci. 39 : 229-231. 